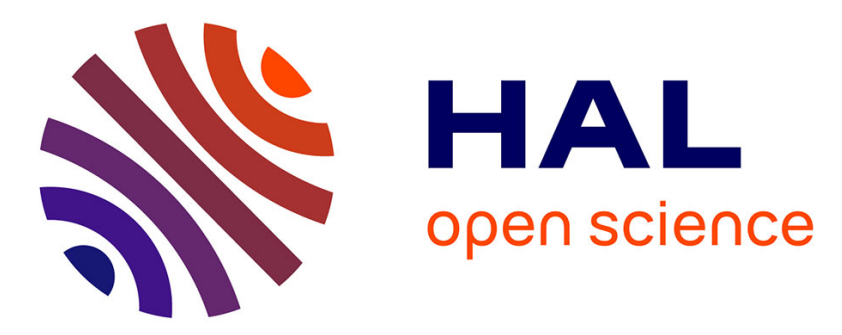

\title{
Acceleration of heavy ions by perpendicular collisionless shocks: Impact of the shock front nonstationarity
}

\author{
Z. W. Yang, Bertrand Lembège, Q. M. Lu
}

\section{To cite this version:}

Z. W. Yang, Bertrand Lembège, Q. M. Lu. Acceleration of heavy ions by perpendicular collisionless shocks: Impact of the shock front nonstationarity. Journal of Geophysical Research Space Physics, 2011, 116 (A10), pp.A10202. 10.1029/2011JA016605 . hal-00628697

\section{HAL Id: hal-00628697 \\ https://hal.science/hal-00628697}

Submitted on 15 Apr 2016

HAL is a multi-disciplinary open access archive for the deposit and dissemination of scientific research documents, whether they are published or not. The documents may come from teaching and research institutions in France or abroad, or from public or private research centers.
L'archive ouverte pluridisciplinaire HAL, est destinée au dépôt et à la diffusion de documents scientifiques de niveau recherche, publiés ou non, émanant des établissements d'enseignement et de recherche français ou étrangers, des laboratoires publics ou privés. 


\title{
Acceleration of heavy ions by perpendicular collisionless shocks: Impact of the shock front nonstationarity
}

\author{
Z. W. Yang, ${ }^{1,2}$ B. Lembège, ${ }^{2}$ and Q. M. Lu ${ }^{1}$ \\ Received 26 February 2010; revised 19 May 2011; accepted 6 July 2011; published 4 October 2011.
}

[1] Both hybrid/full particle simulations and recent experimental results have clearly evidenced that the front of a supercritical quasi-perpendicular shock can be nonstationary. One responsible mechanism proposed for this nonstationarity is the self-reformation of the front itself being due to the accumulation of reflected ions. Important consequences of this nonstationarity are that not only the amplitude but also the spatial scales of fields components at the shock front (ramp and foot) are strongly varying within each cycle of the self-reformation. On the other hand, several studies have been made on the acceleration and heating of heavy ions but most have been restricted to a stationary shock profile only. Herein, one-dimensional test particle simulations based on shock profiles fields produced in PIC simulation are performed in order to investigate the impact of the shock front nonstationarity on heavy ion acceleration ( $\mathrm{He}, \mathrm{O}, \mathrm{Fe})$. Reflection and acceleration mechanisms of heavy ions (with different initial thermal velocities and different charge-mass ratios) interacting with a nonstationary shock front (self-reformation) are analyzed in detail. Present preliminary results show that: (1) the heavy ions suffer both shock drift acceleration (SDA) and shock surfing acceleration (SSA) mechanisms; (2) the fraction of reflected heavy ions increases with initial thermal velocity, charge-mass ratio and decreasing shock front width at both stationary shocks (situation equivalent to fixed shock cases) and nonstationary shocks (situation equivalent to continuously time-evolving shock cases); (3) the shock front nonstationarity (time-evolving shock case) facilitates the reflection of heavy ions; (4) a striking feature is the formation of an injected monoenergetic heavy ions population which persists in the shock front spectrum for different initial thermal velocities and ions species. The impact of the shock front nonstationarity on the heavy ions spectra within the shock front region and the downstream region are detailed separately. Present results are compared with previous experimental analysis and theoretical models of solar energetic particles (SEP) events. The variations of Fe/O spectra in high energy part have been retrieved, and the nonstationary effects of shock front strongly amplify these variations.

Citation: Yang, Z. W., B. Lembège, and Q. M. Lu (2011), Acceleration of heavy ions by perpendicular collisionless shocks: Impact of the shock front nonstationarity, J. Geophys. Res., 116, A10202, doi:10.1029/2011JA016605.

\section{Introduction}

[2] A large body of solar observations in the 1980s 1990s led to the formulation of a standard model, by which solar energetic particle (SEP) events are divided into two categories: gradual and impulsive. According to the two-class paradigm (e.g., review by Reames [1999]), the impulsive events are associated with short duration hard X-ray flares, occur very frequently $(<1000$ events per year during solar maximum), are electron rich, have enhanced heavy ion abundance ratios, and elevated charge states corresponding

\footnotetext{
${ }^{1}$ CAS Key Laboratory of Basic Plasma Physics, School of Earth and Space Sciences, University of Science and Technology of China, Hefei, China.

${ }^{2}$ LATMOS-UVSQ-IPSL-CNRS, Guyancourt, France.

Copyright 2011 by the American Geophysical Union. 0148-0227/11/2011JA016605
}

to hot $<10 \mathrm{MK}$ temperature. In contrast, the gradual events are associated with long duration soft X-ray flares, CMEs, occur at a rate of $<20$ events per year during solar maximum, are proton rich; particle intensities in the interplanetary (IP) space are enhanced for several days, have abundance ratios and charge states typical for the solar corona and solar wind, and ion energy spectra are often power laws with a cut-off (i.e. shoulder) at very high energies. The commonly accepted view of SEP events lead to their classification into either impulsive or gradual depending on the corresponding dominant acceleration mechanism. Particles of gradual events are those in which the overwhelming preponderance of evidence points to acceleration at shocks driven by fast coronal mass ejections (CMEs) [Kahler, 1994; Reames et al., 1997; Reames, 2002]. In contrast, particles of impulsive events are generally ascribed to acceleration at sites associated with flares and 
magnetic reconnection (independent of fast CME-driven shocks) [Hsieh and Simpson, 1970; Reames et al., 1994; Wang et al., 2006]. Moreover, some gradual solar energetic particle events show unusual features at high energies that are typically associated with impulsive events. Proposed reasons for these hybrid events are based on superposed individual impulsive and gradual events, and the seed population typically comprises ions at least from the corona or solar wind (ambient, unheated, coronal material) and from flares (the ions are highly stripped of orbital electrons by the hot environment) [Cohen et al., 2005; Tylka et al., 2005, 2006]. Their studies are based on a large amount of observations by the Advanced Composition Explorer (ACE), Wind, the Interplanetary Monitoring Platform 8 (IMP8), and GEOS missions. The acceleration of compound seed populations by shock in large gradual SEP event has been modeled subsequently [Tylka and Lee, 2006; Sandroos and Vainio, 2007]. In their models, heavy ions as well as protons can be accelerated by shock wave in the absence of a shock front microstructure, and the proposed mechanism is the diffusive shock acceleration (DSA). The authors also realized that additional work was needed to test the limit of the simplified shock geometry hypothesis which was mainly phenomenological. Herein, we propose to analyze the dynamics of heavy ions interacting with selfconsistent shock profiles produced in particle in cell (PIC) simulations. Full PIC simulations including simultaneously different heavy ion species have a high computer cost. Presently, the interaction of these heavy ions is analyzed with test particles technique which has the advantages of relative simplicity and low computer costs. In addition, the combined seed population model created by Tylka and Lee [2006] gives us a motivation to analyze the effect of chargemass ratio and initial thermal velocity on ion acceleration in detail by using test particles approach. Moreover, the use of shock profiles produced in PIC simulation present the advantages of including not only the microstructures of the shock front but also the self-consistent temporal-variation of these structures (nonstationary effects).

[3] The theory of diffusive shock acceleration (DSA) has been intensively used since more than 30 years [Axford et al., 1977; Bell, 1978a, 1978b; Krymsky, 1977; Blandford and Ostriker, 1978; Lee, 1983; Blandford and Eicher, 1987; Webb et al., 1995; Malkov and O'C Drury, 2001; Li et al., 2003; Giacalone, 2004; Zank et al., 2006] and is currently believed to be the most important mechanism of shock acceleration for a variety of space physics environments, for example, planetary bow shocks, interplanetary (IP) shock waves driven outward by CMEs, the heliospheric termination shock (TS), cometary shocks, and shocks associated with supernova remnants (SNR). It is generally thought that DSA should favor the acceleration of heavy ions over lighter ions since the larger gyro-radii ions would be "injected" more easily into the acceleration process. However, a similar theory does not apply at quasi-perpendicular shocks $\left(\theta_{B n}\right.$ $>45^{\circ}$, where $\theta_{B n}$ is the angle between the shock normal and the upstream magnetic field) where the reflected ions return to the shocks almost immediately due to the gyro-motion in the upstream magnetic field. Therefore, other mechanisms such as the shock drift acceleration (SDA) [Hudson, 1965; Webb et al., 1983; Decker and Vlahos, 1985; Decker, 1988; Begelman and Kirk, 1990; Chalov, 2001] and the shock surfing acceleration (SSA) [Sagdeev, 1966; Katsouleas and Dawson, 1983; Zank et al., 1996; Lee et al., 1996; Lipatov et al., 1998; Lee, 1999; Shapiro and Üçer, 2003; Üçer and Shapiro, 2001, 2005] have been proposed and are considered to play important roles on ion acceleration or preacceleration at quasi-perpendicular shocks. Nevertheless, the formation of energetic heavy ions accelerated by quasiperpendicular shocks requires a deeper analysis.

[4] With 1D hybrid simulations, Omidi et al. [1986] have investigated the effect of velocity distribution function of heavy ions $\left(\mathrm{O}^{+}\right)$on the formation and structure of cometary shocks. The results of their study indicate that, at high Mach numbers, the quasi-perpendicular shock structure is highly dependent on the type of the heavy ions $\left(\mathrm{O}^{+}\right)$distribution. Heavy ions from a ring distribution can be reflected back to the upstream region and form a large foot, while heavy ions from a Maxwellian distribution can mostly penetrate the shock without reflection and without affecting the shock structure as much. In above studies, the used spatial resolution is $0.5 c / \omega_{p i}$ (where $c / \omega_{p i}$ is the ion inertial length). As a consequence, the shock profile is stationary as the spatial resolution is not high enough to allow the self-reformation to set up [Hellinger et al., 2002]. The effect of initial thermal velocity of heavy ions on their reflection and acceleration at nonstationary shocks has not been analyzed. Toida and Ohsawa [1997] studied the acceleration mechanisms of four ion species $(\mathrm{H}, \mathrm{He}, \mathrm{O}$ and $\mathrm{Fe})$ at strictly perpendicular shocks by using both theoretical method and PIC simulation. According to their theory, the fraction of reflected ions increases rapidly with their charge-mass ratio. In their simulation, the authors found that all heavy ions pass through the shock front directly and are accelerated by the transverse electric field; the longitudinal electric field has no noticeable impact. The maximum velocity of heavy ions within the shock front is almost independent on their mass. In above studies, the shock front was again stationary, and the impact of charge-mass ratio of heavy ions on their reflection and acceleration at nonstationary shock has not been investigated.

[5] Previous experiments [Morse et al., 1972], observations [Lobzin et al., 2007; Mazelle et al., 2010], hybrid [Hellinger et al., 2002; Yuan et al., 2009] and particle in cell (PIC) simulations [Biskamp and Welter, 1972; Lembège and Dawson, 1987; Lembège and Savoini, 1992; Shimada and Hoshino, 2000; Schmitz et al., 2002; Hada et al., 2003; Scholer et al., 2003; Nishimura et al., 2003; Lee et al., 2004, 2005; Lembège et al., 2009] have revealed that high Mach number, collisionless, shocks are nonstationary. One proposed mechanism responsible for this nonstationarity is the self-reformation of the shock front due to the accumulation of reflected ions at a foot distance from the ramp. This nonstationary process has been well evidenced in $1 \mathrm{D} / 2 \mathrm{D}$ PIC and hybrid simulations, and persists quite well even for realistic mass ratio [Scholer and Matsukiyo, 2004]. Yang et al. [2009a] have investigated the ion acceleration at such nonstationary shocks. However, their study was restricted to protons with Maxwellian and shell distributions, and the impact of shock front reformation on heavy ion acceleration has not been analyzed. Chapman et al. [2005] have investigated the impact of heavy ions (helium) with different density percentages on the shock front self-reformation of perpendicular shocks. Their study indicates that the self- 
Table 1. Upstream Plasma Parameters Defined for PIC Simulations

\begin{tabular}{lccc}
\hline & & Electrons & Ions \\
\hline Thermal velocity & $\tilde{V}_{t h x, y, z}$ & 0.2 & 0.017 \\
Debye length & $\tilde{\lambda}_{D}$ & 0.2 & 0.16 \\
Larmor gyroradius & $\tilde{\rho}_{c}$ & 0.4 & 2.91 \\
Inertia length & $\tilde{c} / \tilde{\omega}_{p}$ & 3.0 & 27.5 \\
Gyro frequency & $\tilde{\Omega}_{c}$ & 0.5 & 0.006 \\
Plasma frequency & $\tilde{\omega}_{p}$ & 1.0 & 0.11 \\
Gyro period & $\tilde{\tau}_{c}$ & 12.55 & 1055.46 \\
Plasma beta & $\tilde{\beta}$ & 0.0355 & 0.0225 \\
\hline
\end{tabular}

reformation is unchanged when the percentage of helium ions is below $10 \%$, but is affected as this percentage is equal to or above $10 \%$. However, the impact of the shock front nonstationarity on the dynamics of heavy ions, and on the resulting heavy ion energy spectra has not been analyzed.

[6] In this paper, we aim to address the following questions by using test particle simulation based on shock profiles produced in one-dimensional PIC simulation: (1) What is the impact of shock front nonstationarity on heavy ion acceleration at a collisionless, supercritical, perpendicular shock? (2) How is this impact according to different initial conditions (varying initial thermal velocity and charge-mass ratio)? (3) What types of acceleration mechanisms (SSA and/or SDA) dominate for these different conditions? (4) What are the consequences of this nonstationarity on heavy ions energy spectra for the different species? This paper is organized as follows. In section 2 , we briefly describe the numerical model. Sections 3 and 4 present the simulation results for (stationary) fixed shock profiles (selected at given times), and for time-evolving shock (nonstationary) profiles, respectively. In section 5, we discuss and compare our results with those of previous papers. The main conclusions are summarized in section 6 .

\section{Simulation Model}

[7] Herein, we implement a combination of a 1-D PIC simulation in order to model the fields of a self-consistent (nonstationary) perpendicular shock profile and a test particle simulation to follow the dynamics of a large number of energetic heavy ions. First, we have performed a onedimensional PIC simulation similar to that of previous works [Lembège and Dawson, 1987; Lembège and Savoini, 1992], and the shock is initiated by a magnetic piston (applied current pulse). Plasma and shock conditions are similar to those used by Hada et al. [2003] and Yang et al. [2009a]. The shock geometry is defined in the upstream frame: the shock propagates along the $x$ direction and an upstream magnetic field $\tilde{B}_{o}$ is applied along the $z$ direction. All dimensionless quantities are indicated by a tilde " $\sim$ " and are normalized as follows. The spatial coordinate is $\tilde{x}=x / \Delta$; velocity $\tilde{\nu}=\nu / \omega_{p e} \Delta$; time $\tilde{t}=\omega_{p e} t$, electric field $\tilde{E}=e E /$ $m_{e} \omega_{p e}{ }^{2} \Delta$; magnetic field $\tilde{B}=e B / m_{e} \omega_{p e}{ }^{2} \Delta$. The parameters $\Delta, \omega_{p e}, m_{e}$ and $e$ are, respectively, the numerical grid size, the electron plasma frequency, the electron mass and the electric charge. All basic parameters are as follows: the size of the plasma simulation box $L_{x}=4096$; velocity of light $\tilde{c}=$ 3 , and mass ratio of proton and electron $M_{i} / m_{e}=84$. Initially, the particle density is $n_{i}=n_{e}=50$ in each grid. The electron/ion temperature ratio $T_{e} / T_{i}=1.58\left(\right.$ low $\left.\beta_{i}\right)$ is chosen in order to investigate the particle acceleration at a reforming shock and to keep a certain coherency with previous PIC simulations [Hada et al., 2003; Yang et al., 2009a]. The ambient magnetic field is $\left|\tilde{B}_{o}\right|=1.5$. The shock has an averaged Alfvénic Mach number, $M_{A}=5.2$ where $M_{A}=$ $V_{\text {shock }} / V_{A}$ is determined in the upstream plasma (i.e. simulation) frame; the Alfvén velocity $\tilde{V}_{A}$ is equal to 0.16 . For these initial conditions, the plasma parameters are summarized in Table 1 for both electrons and protons, the Larmor gyro-radius in Table 1 is calculated based on thermal velocity. Table 2 summarizes the plasma parameters used for different heavy ions in the test particle simulations.

[8] Second, we integrate the full motion of test-particle heavy ions in the electric and magnetic fields obtained in the above PIC simulation. Heavy ions are described with Maxwellian velocity distribution. The parametric analysis is performed with different thermal velocities $\tilde{V}_{t h i}$ (from $0.1 \tilde{V}_{\text {thp }}$ to $30 \tilde{V}_{t h p}$, where $\tilde{V}_{\text {thp }}$ is the proton thermal velocity in our PIC simulation) and different charge-mass ratios $\mathrm{Q} / \mathrm{M}$ (from 1 to $7 / 16$ ). Four species of ions $\left(\mathrm{H}^{+},{ }^{3} \mathrm{H}^{2+},{ }^{4} \mathrm{He}^{2+}\right.$ or $\mathrm{O}^{8+}$, and $\mathrm{O}^{7+}$ ) are considered here, and their charge-mass ratios are $1,2 / 3,1 / 2$ and $7 / 16$, respectively. ${ }^{4} \mathrm{He}^{2+}$ and $\mathrm{O}^{7+}$ are typical corona seed populations, and ${ }^{3} \mathrm{H}^{2+}$ and $\mathrm{O}^{8+}$ are typical flare seed populations [Tylka and Lee, 2006]. Initially, these test particles are uniformly distributed in the far upstream (50 particles per cell). For a given time, a shock profile is selected and interacts with test particles during its propagation. Our test particle simulations are separated into two parts. In the first part (fixed shock), we choose three typical shock profiles at three different times within one selfreformation cycle, and we analyze separately the dynamics of heavy ions interacting with these stationary-like shock profiles. Here, the propagating shock is injected with an instantaneous velocity equal to that directly measured in the PIC simulation; note that an instantaneous Mach number may differ from the value averaged over one full selfreformation cycle $\left(M_{A}=5.24\right)$. In the second part (timeevolving shock), we investigate the dynamics of heavy ions interacting with a shock continuously time-evolving during its self-reformation. The test particles encounter the shock front continuously, and the simulation time range covers about five reformation cycles of the shock front.

[9] We note that in our model the test particle simulation of heavy ions is not self-consistent. The heavy ion scale shock structure, which may be important, is neglected here. Solving the whole problem of heavy ions with a high (or realistic) mass value and full PIC simulations for quite different heavy ions species is computationally very expensive. Our present approach results from a compromise based on the results of Chapman et al. [2005] which indicate that the characteristic features of the shock front self-reformation is not affected by the presence of heavy ions provided their

Table 2. Parameters for Different Ion Species (Figures 6, 7, 8, and 13), for the Case $\tilde{V}_{t h i}=5 \tilde{V}_{t h p}$

\begin{tabular}{lccccc}
\hline & & $\mathrm{H}^{+}$ & ${ }^{3} \mathrm{He}^{2+}$ & ${ }^{4} \mathrm{He}^{2+}$ or $\mathrm{O}^{8+}$ & $\mathrm{O}^{7+}$ \\
\hline Thermal velocity & $\tilde{V}_{t h i x, y, z}$ & 0.085 & 0.085 & 0.085 & 0.085 \\
Larmor gyroradius & $\tilde{\Omega}_{c i}$ & 14 & 21 & 28 & 33 \\
Gyro frequency & $\Omega_{c i}$ & 0.006 & 0.004 & 0.003 & 0.0026 \\
Gyro period & $\tilde{\tau}_{c i}$ & 1056 & 1583 & 2111 & 2413 \\
Plasma beta & $\tilde{\beta}_{i}$ & 0.55 & 0.825 & 1.1 & 1.26 \\
\hline
\end{tabular}



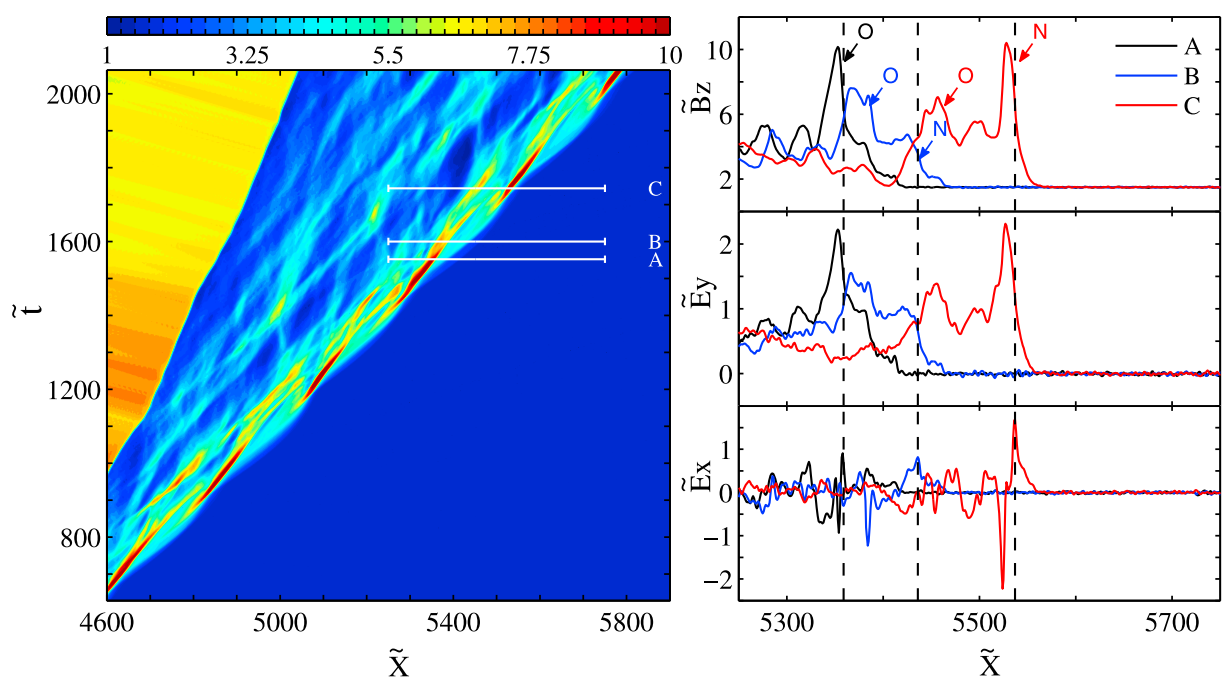

Figure 1. (left) Time evolution of $\tilde{B}_{z}$ versus time. (right) Main field components $\tilde{B}_{z}, \tilde{E}_{y}$ and $\tilde{E}_{x}$ of three typical shock profiles chosen at $\tilde{t}=1552$ (black), 1600 (blue) and 1744 (red), within one self-reformation cycle. Black dashed vertical lines indicate the main ramp locations at these different shock profiles, where the electrostatic field $\tilde{E}_{x}$ is maximum. These three profiles are denoted by A, B and C in Figure 1 (left), respectively [from Yang et al., 2009a].

percentage is below a certain threshold (less than $10 \%$ for Helium); then, the microstructures of the shock front and its nonstationarity is self-consistently present and mainly driven by protons. Our present simulations apply mainly to this case. In addition, our approach presents the advantage of relative simplicity, of low computer cost and allows us to perform a detailed parametric analysis and high statistical study necessary for computing the energy spectra for the different ion species.

\section{Simulation Results: Fixed Shock Profiles}

[10] The nonstationarity of the supercritical perpendicular shock is illustrated in Figure 1 (left), which shows the time evolution of the magnetic field $\widetilde{B}_{z}$ from $\tilde{t}=628$ to 2064 . At $\tilde{t}=650$, the shock ramp (the old ramp) is at about $\tilde{X}=4615$. Later at about $\tilde{t}=800$, the relatively high percentage of reflected protons accumulates in the foot so that the foot amplitude keeps increasing in time until its edge is high enough to become a new ramp. At about $\tilde{t}=930$, the amplitude of the new ramp located around $\tilde{X}=4850$ exceeds that of the old ramp. Simultaneously, the old ramp downstream of the new ramp becomes weaker. The nonstationary shock front is characterized by a self-reformation process with a cyclic period about $256 \tilde{\omega}_{p e}^{-1} \approx 1.5 \tilde{\Omega}_{c i}^{-1}$. This shock front reformation has been interpreted as a coupling between the "incoming" and "reflecting" protons [Hada et al., 2003]. In this paper, we focus our attention to the impact of the shock front self-reformation on heavy ion acceleration.

[11] In this section, three typical shock profiles (A, B and C) are selected at late times within the fifth self-reformation cycle (from $\tilde{t}=1488$ to 1744 ), and are shown in Figure 1 (right). Profiles A, B and C represent the snapshots of $\tilde{B}_{z}$, $\tilde{E}_{y}$ and $\tilde{E}_{x}$ at $\tilde{t}=1552,1600$ and 1744 , respectively. In profile $\mathrm{A}$, the shock front includes a ramp and a foot, and the position of the ramp is denoted by "O" (old) ramp. In profile $\mathrm{B}$, the amplitude of the old ramp has decreased. Simultaneously, the foot amplitude (and its width) increases and reaches a value at least $50 \%$ of that of the old one and becomes a new ramp "N". In profile $\mathrm{C}$, the amplitude of the new ramp has already overcome the old one. For profiles $\mathrm{B}$ and $\mathrm{C}$, the reference ramp used herein is the main new ramp ("N"), which has larger $\tilde{E}_{x}$ than the old one. The ramp positions for profile $\mathrm{A}, \mathrm{B}$ and $\mathrm{C}$ are $\tilde{X}=5359,5436$ and 5537, and their corresponding instantaneous propagating velocities $\tilde{V}_{\text {shock }}$ along $x$ direction are $51.5 \tilde{V}_{\text {thp }}, 58.8 \tilde{V}_{\text {thp }}$ and $36.8 \tilde{V}_{t h p}$. The shock front widths of profile A, B and C (including foot and ramp) equals to $2.4 \tilde{c} / \tilde{\omega}_{p i}, 3.6 \tilde{c} / \tilde{\omega}_{p i}$ and $1.3 \tilde{c} / \tilde{\omega}_{p i}$, which are in proton scale. Corresponding ramp width is about $2 \tilde{c} / \tilde{\omega}_{p e}, 23 \tilde{c} / \tilde{\omega}_{p e}$ and $3 \tilde{c} / \tilde{\omega}_{p e}$, respectively. These fixed shock profiles will allow us to address two important issues. One is the effect of initial thermal velocity of heavy ions on their reflection, acceleration and energy spectrum, and the other is the impact of the charge-mass ratio.

[12] At the beginning of test particle simulation, we initially give each test particle an identification index. At each time step, the new incoming ions are divided into three groups: the shock drift accelerated (SDA) ions, the shock surfing accelerated (SSA) ions and the directly transmitted (DT) ions, following the same selection process as that used by Yang et al. [2009a]. Simultaneously, their index values are changed to 1 (for SSA), 2(for SDA) and 3 (for DT), respectively. As soon as the new index value is affected, it will stay unchanged forever, and the count of each population continues. For a given group (SSA, SDA or DT ions), the energy spectrum is calculated at any moment during the test particle simulation. In present section 3 (fixed shock profiles), each test particle simulation stops at an ending time which is defined as the time before first heavy ions approach the piston far downstream behind the shock front. 

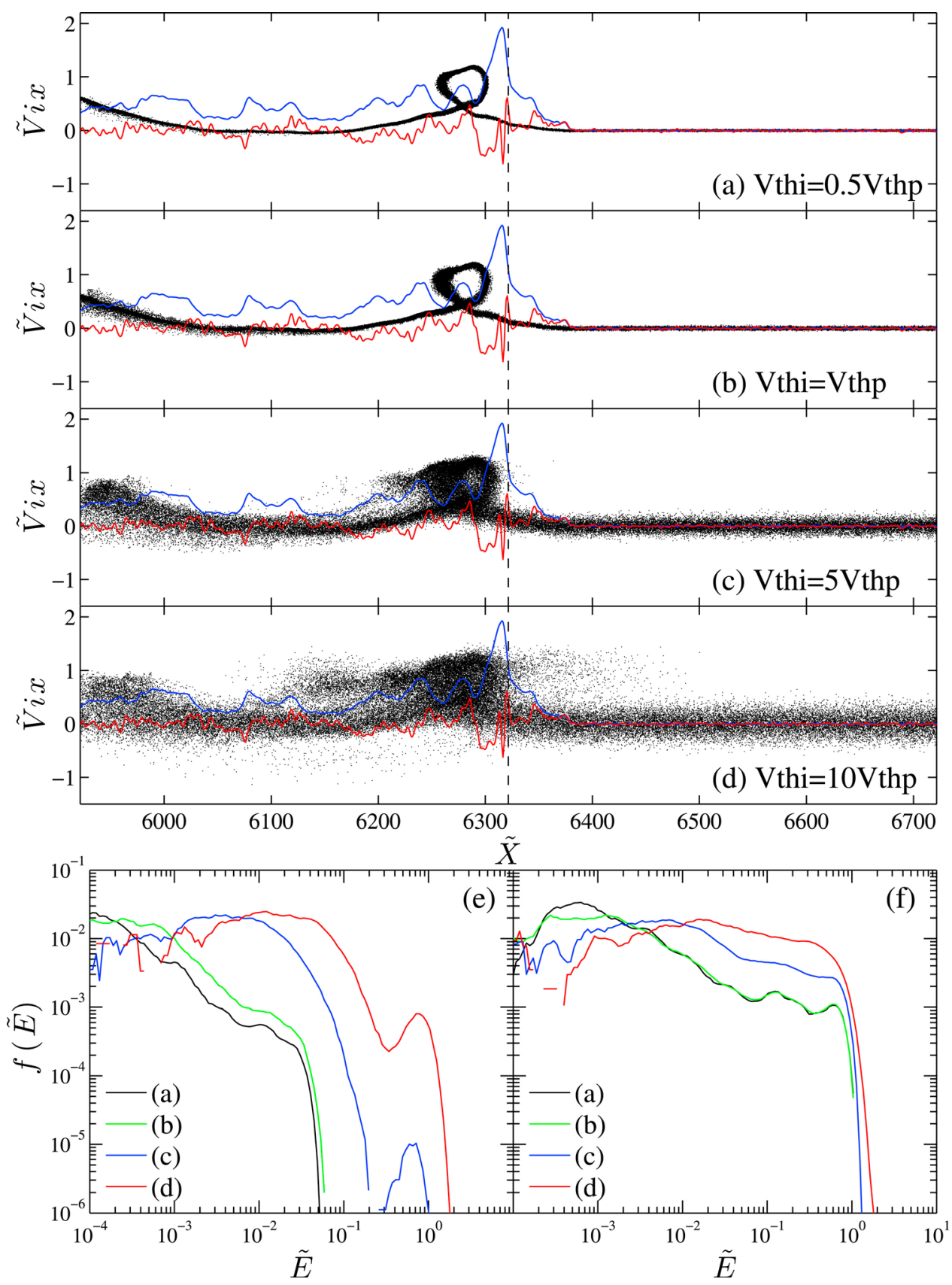

Figure 2. (a-d) The phase space plots $\left(\tilde{V}_{i x}\right.$ versus $\left.\tilde{X}\right)$ of helium ions ${ }^{4} \mathrm{He}^{2+}$ with different initial Maxwellian thermal velocities for profile A chosen at time $\tilde{t}=1552$. The magnetic field $\tilde{B}_{z}$ (blue) and electrostatic field $\tilde{E}_{x}$ (red) components are also shown for reference in each plot. (e and f) The corresponding normalized energy spectra measured within the shock front and the downstream region obtained from Figures 2a-2d; each case (Figures 2a-2d) of Figures $2 \mathrm{e}$ and $2 \mathrm{f}$ is identified by different colors.

Since we select shock profiles at late times of the PIC simulation, heavy ions have enough time to interact with the shock front and the downstream structures which include the reminiscent signatures of the previous self-reformations. Then, two spectra are considered for a given profile: (1) the shock front spectrum includes all ions located between the upstream edge of the foot (where local $\tilde{B}_{z}$ exceeds a threshold (here $\tilde{B}_{o}+0.1$ ) chosen such that it is slightly larger than the maximum amplitude of upstream $\tilde{B}_{z}$ field turbu- lence) and the ramp (defined at the location of the maximum $\tilde{E}_{x}$ peak); (2) the downstream spectrum includes all ions located between this same ramp and the far downstream limit (reached by the last heavy ions penetrating further downstream); this limit can vary according to the initial $\tilde{V}_{t h i}$ and the $\mathrm{Q} / \mathrm{M}$ ratio. Same procedure applies to other profiles at $\tilde{t}=1600$ (profile B) and $\tilde{t}=1744$ (profile C). The ramp location is indicated by a vertical dashed line in Figures 2, 3, 4, 5, 6, 7, and 8 . 

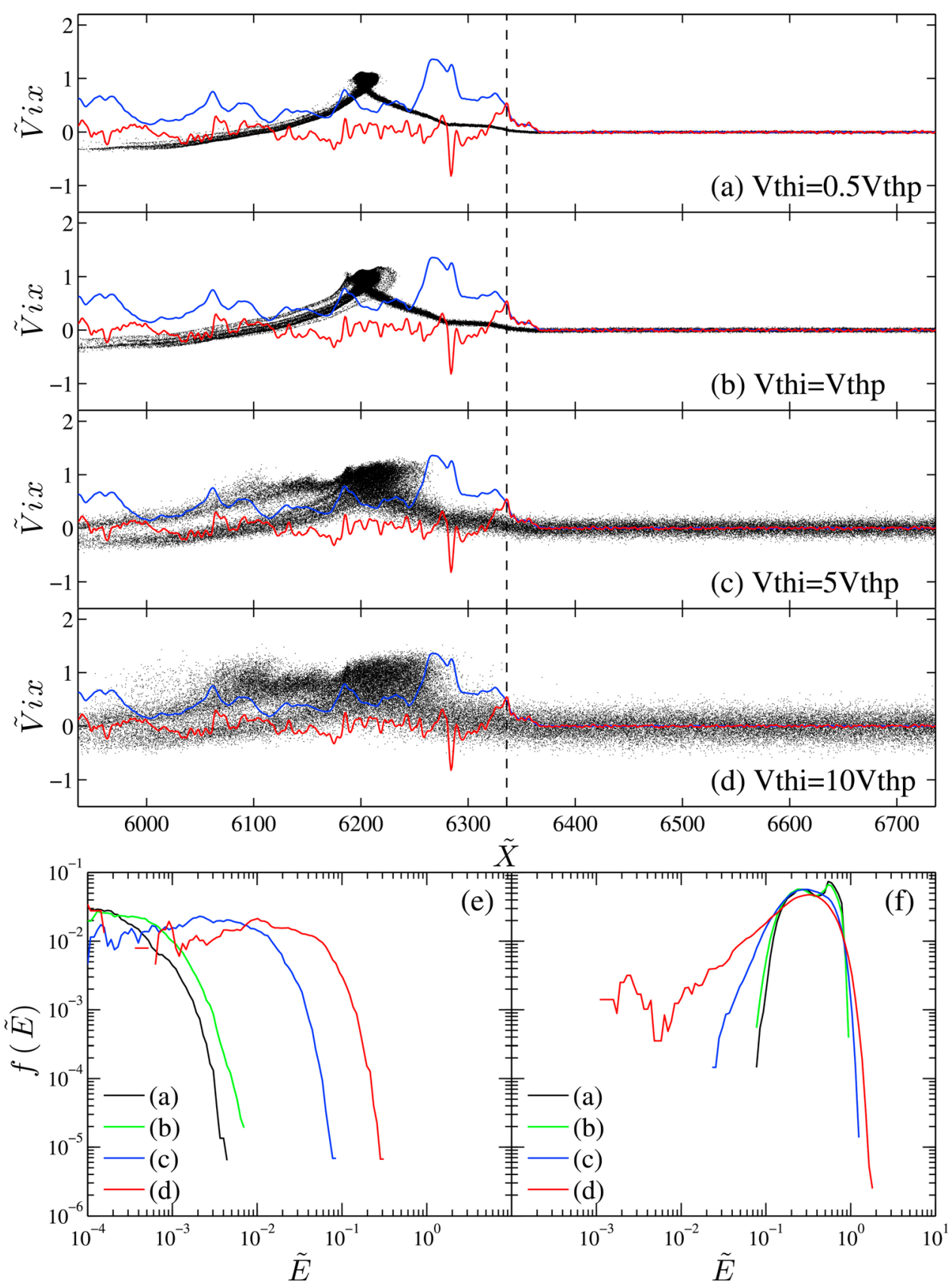

Figure 3. Similar plots as Figure 2 for shock profile B at $\tilde{t}=1600$.

\subsection{Impact of Initial Thermal Energy}

[13] Herein, we focus on the effect of initial thermal velocity on heavy ion (helium ${ }^{4} \mathrm{He}^{2+}$ ) reflection and acceleration at different shock profiles. For profile A, phase space plots are shown in Figures 2a-2d. Maxwellian distributions of helium ${ }^{4} \mathrm{He}^{2+}$ are considered with different initial thermal velocities $0.5 \tilde{V}_{t h p}$ (Figure 2a), $\tilde{V}_{t h p}$ (Figure 2b), $5 \tilde{V}_{t h p}$ (Figure 2c), and $10 \tilde{V}_{\text {thp }}$ (Figure 2d). Main magnetic field and longitudinal electric field are also shown for reference in each panel. Results show that (1) helium ions with low initial thermal velocity cannot be reflected by the shock ramp (Figures $2 a$ and $2 b$ ), (2) the center location of their rotation/vortex in phase space is always behind the overshoot (defined by protons in PIC simulations), because of their inertia stronger than that of protons. These vortices keep some coherency for low thermal velocity (Figures 2a and $2 b$ ) but become more diffuse for high thermal velocity cases (Figures 2c and 2d); (3) once the initial thermal velocity of ${ }^{4} \mathrm{He}^{2+}$ particles exceeds a threshold $\left(\tilde{V}_{t h p}\right)$, a fraction of reflected ions emerges and their density rapidly increases with thermal velocity. Single trajectories and energization processes of those reflected ions will be analyzed in Figure 9. Figure 2e shows the corresponding shock front spectra versus $\tilde{E}$ (here, $\tilde{E}=\tilde{E}_{k} / \tilde{M}_{i}, \tilde{E}_{k}, \tilde{E}_{k}$ is the 

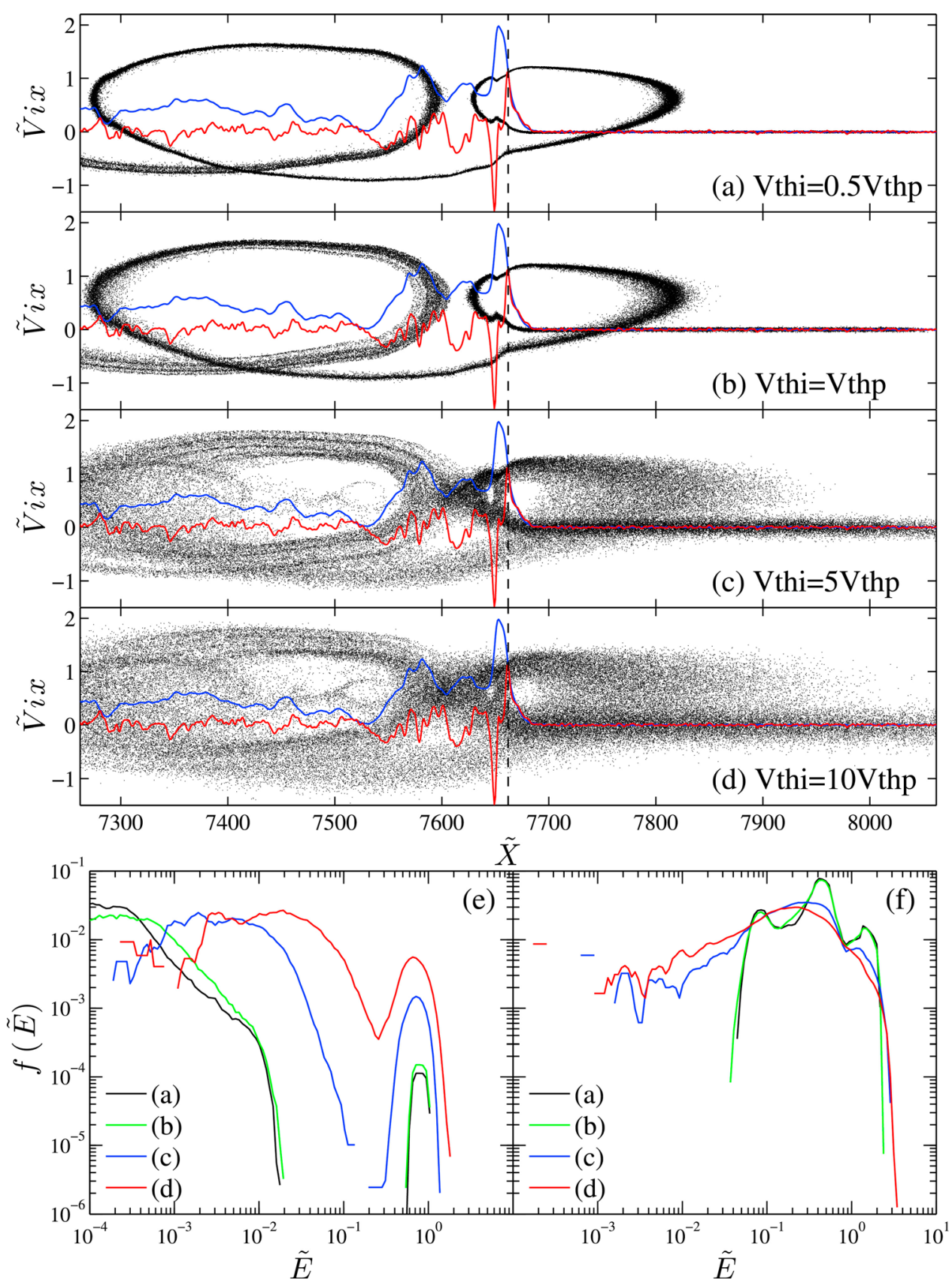

Figure 4. Similar plots as Figure 2 for shock profile C at $\tilde{t}=1744$.

dimensionless kinetic energy, $\tilde{M}_{i}$ is the dimensionless mass ratio normalized with respect to the electron mass for different ion species; e.g. $M_{i} / m_{e}=84,252,336$ and 1344 for $\mathrm{H},{ }^{3} \mathrm{He},{ }^{4} \mathrm{He}$ and $\mathrm{O}$ components, respectively). For high $\tilde{V}_{t h p}$, the origin of the bump around 0.7 (Figures 2c and 2d) correspond to particle reflection. Figure $2 \mathrm{f}$ shows the corresponding downstream spectra of helium ions. Those spectra exhibit a power law form $\left(\tilde{E}^{-k}\right)$ in the middle energy part $(0.005 \sim 0.5)$, with a characteristic index $k$ weakly varying between $0.5<k<0.6$. A distinct maximum energy cut off occurs in the high energy range (above $\sim 0.5$ ) for each case, which only slightly increases with $\tilde{V}_{t h p}$, within the range 1 2.
[14] Figures 3a-3d shows the phase space plots of ${ }^{4} \mathrm{He}^{2+}$ particles for profile B. Shock profile B exhibits a lower (overshoot) and a broaden shock front (including ramp and foot), and represents an easier barrier to pass through. Indeed, all incident ions are directly transmitted downstream whatever $\tilde{V}_{t h i}$ is. The location of the vortex center is largely shifted further downstream (as compared to profile A) but stays unchanged with $\tilde{V}_{t h i}$. However, important particle diffusion again takes place, as $\tilde{V}_{t h i}$ increases.

[15] Figures $3 \mathrm{e}$ and $3 \mathrm{f}$ represent their shock front spectra and downstream spectra, respectively. The main core of shock front spectra corresponds to upstream ions and can be used as reference (Figure 3e); no high energy bump is 


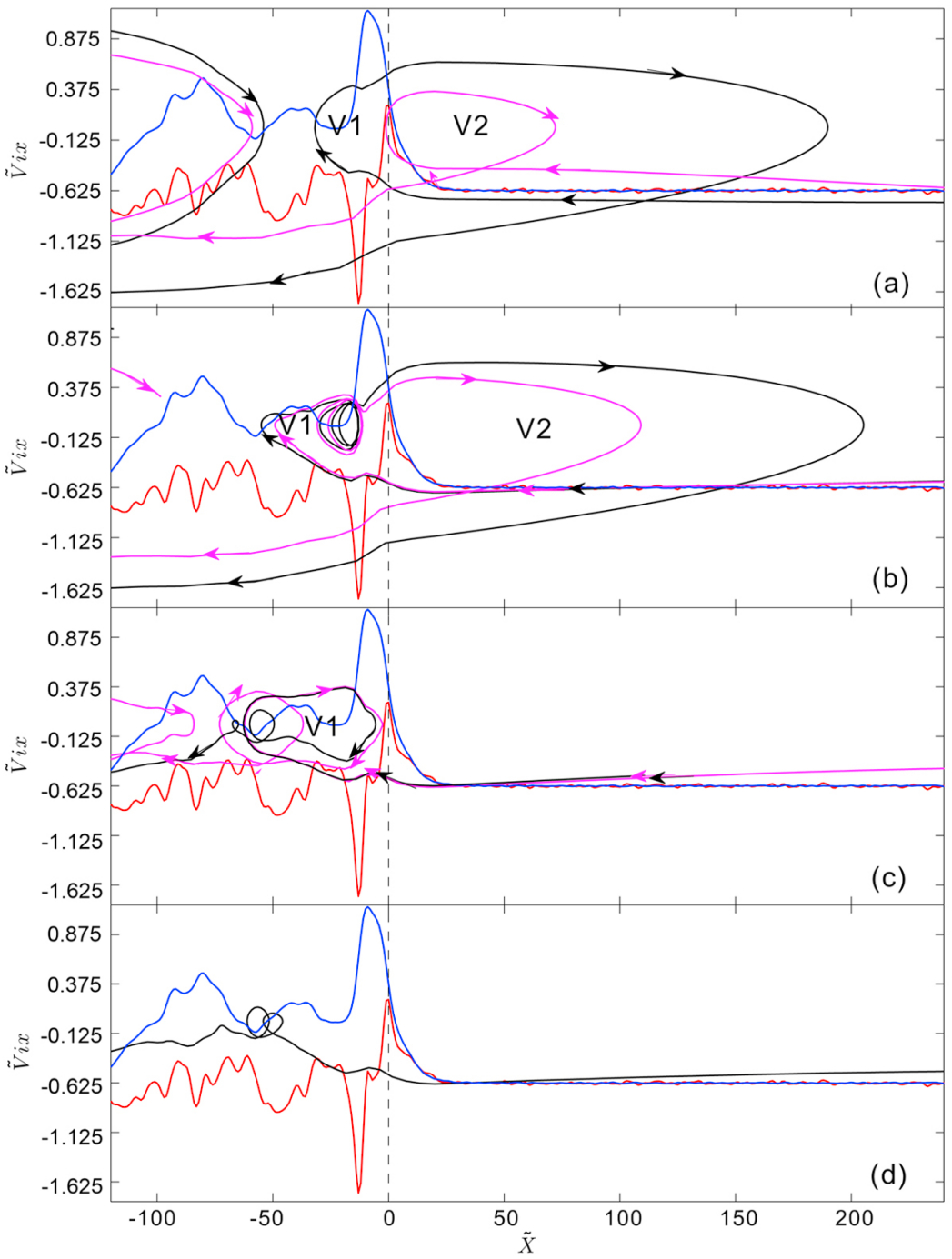

Figure 5. (a-d) Different types of typical phase space trajectories of helium ions ${ }^{4} \mathrm{He}^{2+}$ selected from Figure 4c $\left(\tilde{V}_{t h i}=5 \tilde{V}_{t h p}\right)$, and shown in the shock rest frame. "V1" and "V2" indicate the vortices in phase space formed, respectively, just behind the overshoot and at the ramp (see Figure 4). Trajectories are shown in different panels for the purpose of clarity. The vertical dashed line denotes the shock ramp location. Profiles of $\tilde{B}_{z}$ (blue) and $\tilde{E}_{x}$ (red) fields components are also shown for reference.

evidenced since no ions are reflected. In both subplots, the spectra have Maxwellian-like form (no power law features). The maximum energy of the shock front spectrum corresponds only to that of the upstream $\tilde{V}_{t h i}$. For downstream spectra, the range of the bump is relatively unchanged (0.09 2 in Figure 3f) with $\tilde{V}_{t h i}$, as compared to those obtained in the reference cases $(0.004 \sim 0.2$ Figure $3 \mathrm{e})$. In addition, a noticeable downstream heating is evidenced by the broadening of the spectrum which becomes larger as $\widetilde{V}_{t h i}$ increases (Figure 3f).

[16] In contrast, at profile $C$, the shock ramp is very steep and the amplitudes of both longitudinal electric field $\widetilde{E}_{x}$ and magnetic field are very strong. Then, the cross-shock potential strongly increases (not shown here) within the narrow shock front, and a high fraction of reflected ions is expected. This is confirmed in the phase space plots of ${ }^{4} \mathrm{He}^{2+}$ particles (Figure 4), where a noticeable percentage of reflected ${ }^{4} \mathrm{He}^{2+}$ ions is evidenced whatever $\tilde{V}_{t h i}$ is.
[17] For low $\tilde{V}_{t h i}\left(<\tilde{V}_{t h p}\right)$, the motion of reflected ions stays very coherent even further downstream (double vortices in Figure 3a) and corresponds to a large ion ring population in perpendicular velocity space (not shown here).

[18] However, for high $\tilde{V}_{t h i}\left(>\widetilde{V}_{t h p}\right)$, the reflected ions become more much diffuse from this large vortex structure. Main features are: first, the spatial extension of the reflected ions (due to this strong diffusion) strongly increases upstream as $\tilde{V}_{t h i}$ increases. Second, the locations of the large multiple vortex centers stay unchanged whatever $\tilde{V}_{t h i}$ is. Third, those vortices are fed by at least four kinds of ions as evidenced by analyzing time trajectories of typical ${ }^{4} \mathrm{He}^{2+}$ ions shown in Figure 5; these ions are selected from Figure 4c. A deeper look of Figure $4 \mathrm{c}$ evidences that the first vortex presents an intricate structure (" $\infty$ " shape) around the shock front $7630<\tilde{x}<7700$, which can be analyzed thanks to these time trajectories. The four kinds of heavy ions correspond to: 

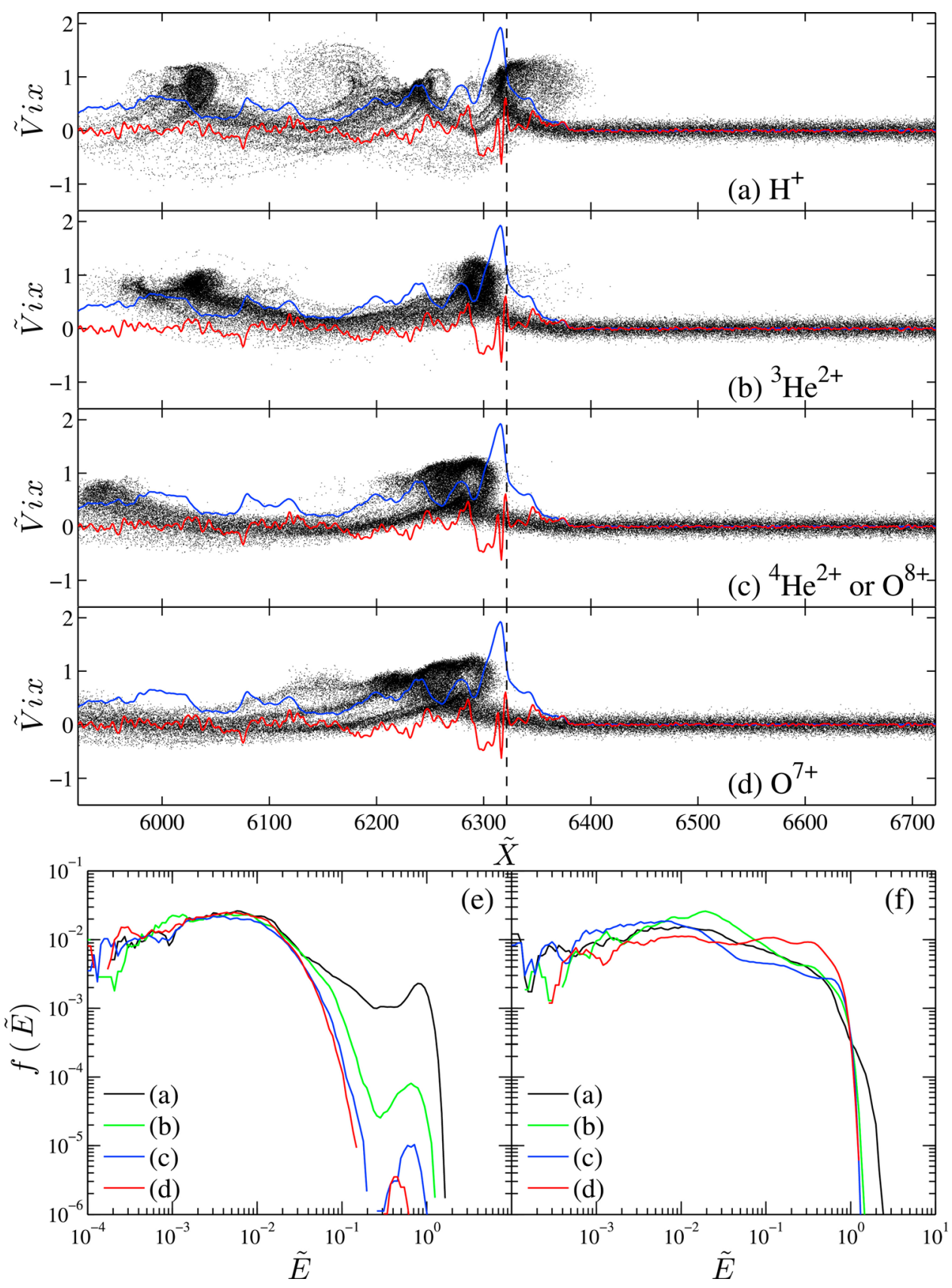

Figure 6. (a-d) The phase space plots $\left(\tilde{V}_{i x}\right.$ versus $\left.\tilde{X}\right)$ of different ion species with the same initial thermal speed $\left(\tilde{V}_{t h i}=5 \tilde{V}_{t h p}\right)$ obtained with profile A at time $\tilde{t}=1552$. The magnetic field $\tilde{B}_{z}$ (blue) and electrostatic field $\tilde{E}_{x}$ (red) components are also shown for reference in each plot. (e and f) The corresponding normalized energy spectra measured within the shock front and the downstream region obtained from Figures 6a-6d; each case (Figures 6a-6d) of Figures 6e and $6 \mathrm{f}$ is identified by different colors.

[19] 1. Ions which are reflected at the ramp (vertical dashed line) and then go downstream (vortex V2 in Figure 5a). Its behavior is similar to proton trajectory.

[20] 2. Ions which are directly transmitted through the ramp but are trapped by the bipolar $\tilde{E}_{x}$ structure in the vicinity of overshoot, are expelled upstream on a large Larmor orbit which forces these to go back downstream (Figure $5 b$ ). The ion which suffers more bounces ( 3 bounces for vortex V1 in Figure 5b) is expelled upstream on a larger orbit (and reach a further part of the upstream region during its gyration) than the ion suffering less bounces ( 2 bounces for vortex V2 in Figure 5b) and even only one bounce (ion V1 in Figure 5a). Ion V1 of Figure 5a has similar behavior as those of lower $\tilde{V}_{t h i}$ (Figure $4 \mathrm{a}$ ).

[21] 3. Ions which penetrate downstream but are reflected behind the overshoot, more precisely between the old and the new ramps (vortex V1 in Figure 5c). The trajectories and energization of these ions are similar to that of protons, 

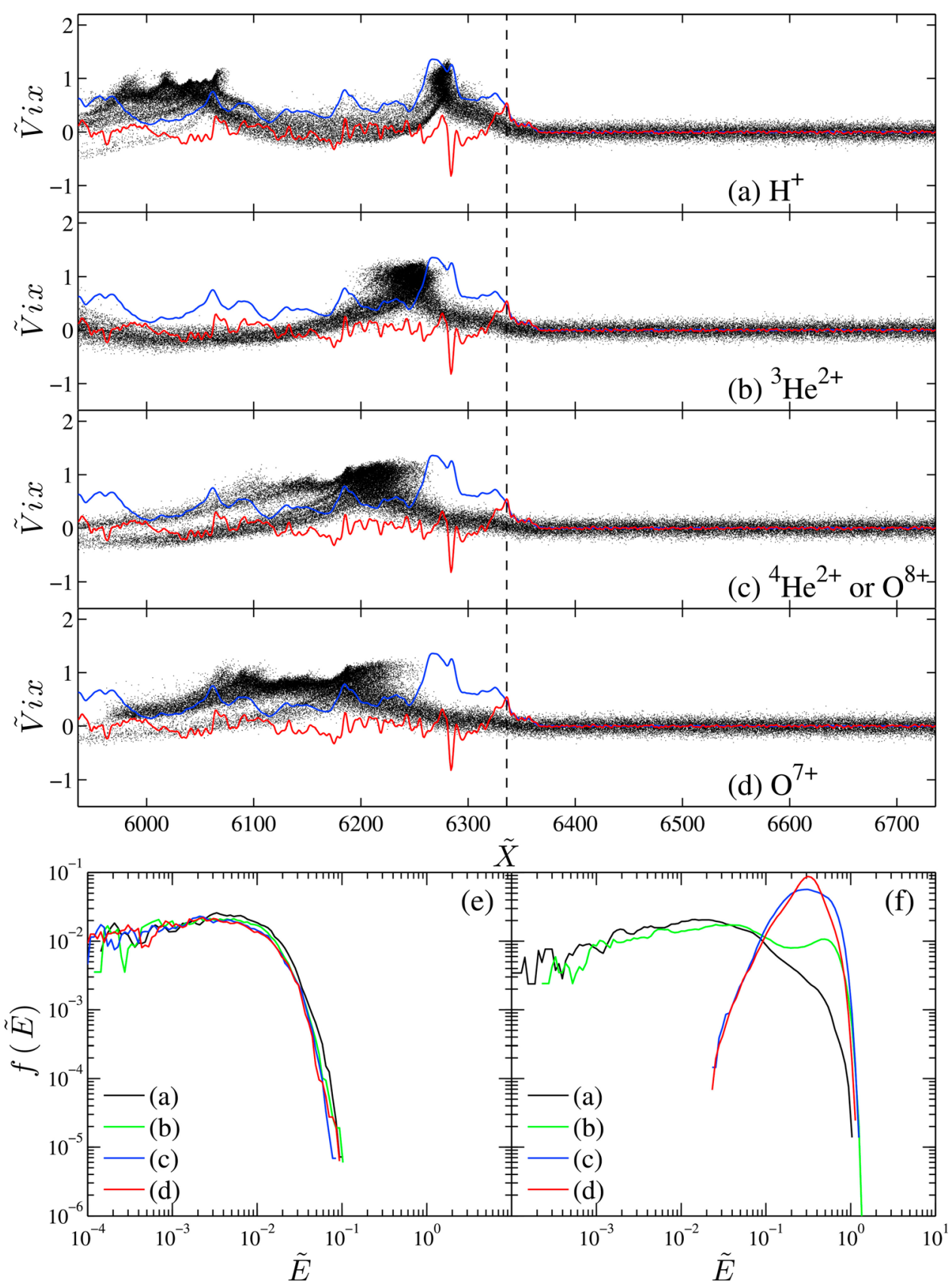

Figure 7. Similar plots as Figure 6 for shock profile B at $\tilde{t}=1600$.

which have already been mentioned in Figure 7 of previous paper [Yang et al. 2009a].

[22] 4. Ions which are directly transmitted through the whole shock front and succeed to penetrate far downstream without any reflection (Figure $5 \mathrm{~d}$ ).

[23] 5. The " $\infty$ " vortex shape observed around the shock front $7630<\tilde{x}<7700$ of Figure 4c, appears as being composed with different types of ion trajectories illustrated by vortices V1 and V2 in Figures 5a-5c.

[24] The full understanding of these different trajectories requires a detailed analysis which is out of scope of the present paper and is left for a further work. The shock front spectra of helium particles shown in Figure 4e allow stres- sing the following striking features: a bump is clearly formed in the high energy range whatever $\tilde{V}_{t h i}$ is. This bump corresponds to the formation of a monoenergetic reflected ${ }^{4} \mathrm{He}^{2+}$ population centered around a high energy value which stays unchanged with $\tilde{V}_{t h i}$, and forms an injected population. However, the fraction of reflected ions (and the width of its spectrum i.e. its heating) strongly increases with $\tilde{V}_{t h i}$. Figure $4 \mathrm{f}$ shows downstream spectra of helium particles. Surprisingly, the spectrum exhibits a strong change from a Maxwellian-like (weak $\tilde{V}_{t h i}$ ) to a power law shape (strong $\tilde{V}_{t h i}$ ). For high $\tilde{V}_{t h i}$, the power law index approximately equals to 1.5 in the middle energy part $(0.3 \sim 2)$, which corresponds to a much steeper spectrum than those 

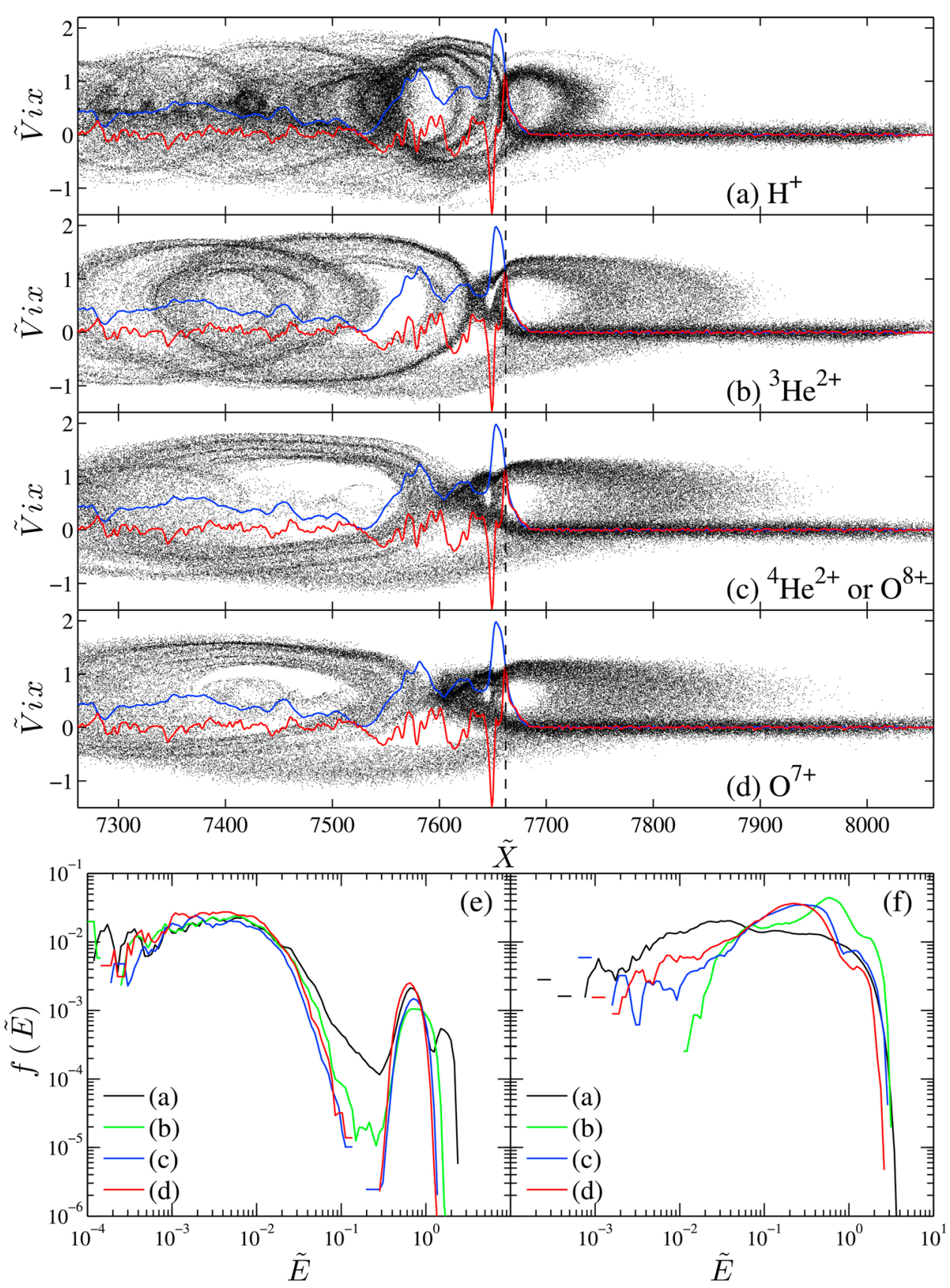

Figure 8. Similar plots as Figure 6 for shock profile C at $\tilde{t}=1744$.

measured in Figure 2. The maximum energy of the spectrum weakly increases (from 2 to 3.1 ) with $\tilde{V}_{t h i}$.

[25] In summary, a very strong contrast is observed in the dynamics of heavy ions with percentage of reflection varying from $0 \%$ to $100 \%$ between profiles $\mathrm{A}$ and $\mathrm{C}$, leading to a strong impact on corresponding shock front and downstream spectra (transition from Maxwellian to power law shapes and formation of monoenergetic population). The maximum energy of downstream spectrum not only increases with initial $\tilde{V}_{t h i}$, but also depends on the shock front profile. In other words, the shock front self-reformation plays the role of a natural filter. All high initial thermal velocity cases are accompanied by diffusion, but the strength of this diffusion strongly depends of the concerned shock profile. In contrast, the location of the heavy ions vortex formed around the shock transition region is independent on the initial thermal velocity.

\subsection{Impact of Charge-Mass Ratio}

[26] Herein, we focus our attention to the impact of charge-mass ratio $(\mathrm{Q} / \mathrm{M})$ on heavy ion acceleration at the same selected shock profiles. All ion species initially have a Maxwellian distribution with the same thermal velocity $\tilde{V}_{t h i}=5 \tilde{V}_{t h p}$ (where the subscript "i" identifies the different ion species). Initial plasma parameters corresponding to the different heavy ions species are summarized in Table 2 . 

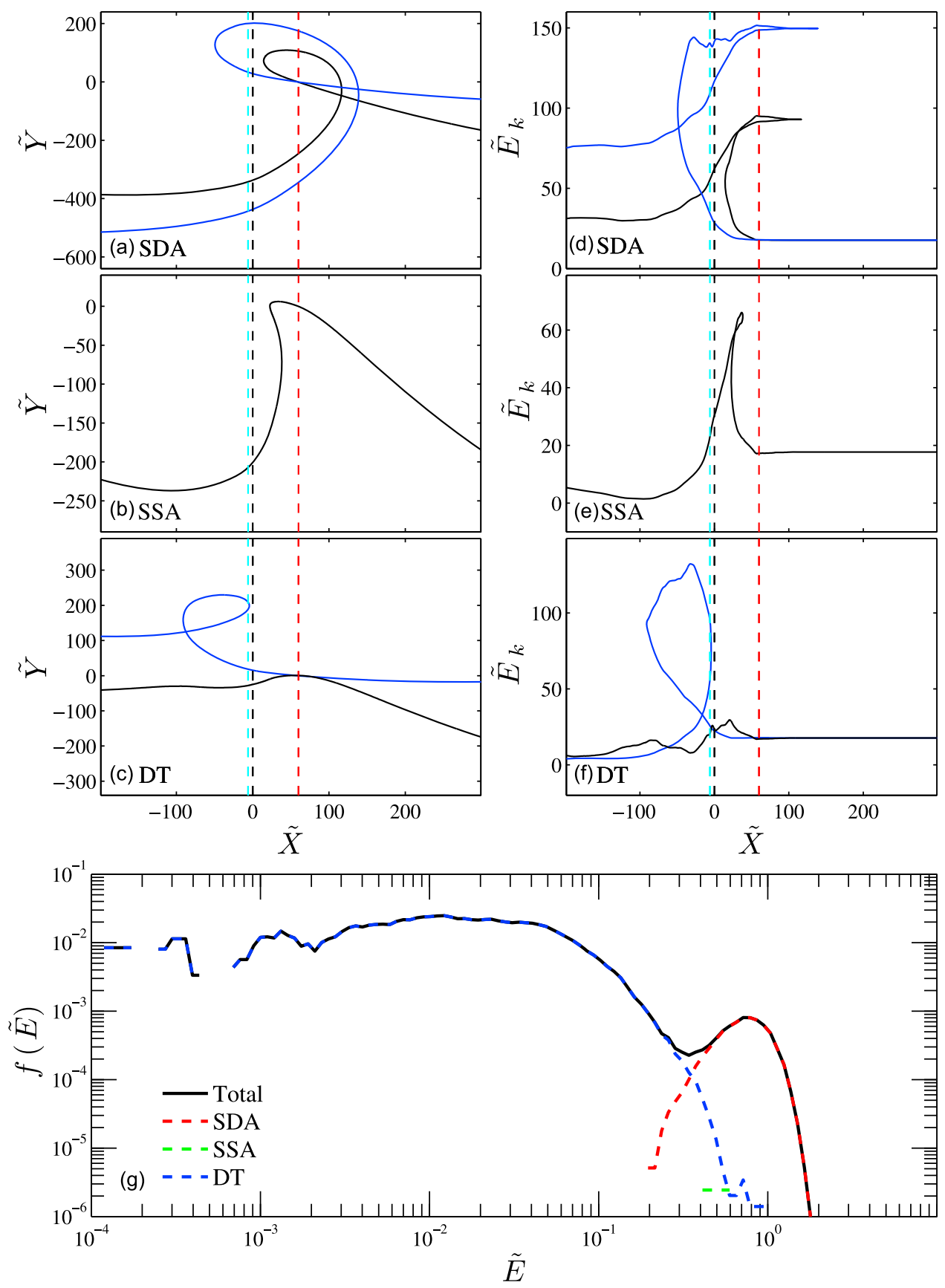

Figure 9. (a-c) Three typical $(\tilde{X}-\tilde{Y})$ trajectories of ${ }^{4} \mathrm{He}^{2+}$ ions suffering respectively SDA, SSA and DT mechanisms at the same shock profile A measured in the shock frame and corresponding to Figure $2 \mathrm{~d}$ (where $\tilde{V}_{t h i}=10 \tilde{V}_{t h p}$ ). The upstream edge of the foot, the ramp and overshoot locations are also shown for reference by red, black and cyan vertical dashed lines respectively. (d-f) Corresponding kinetic energy $\tilde{E}_{k}$ versus $X$ are shown. (g) The different normalized energy spectra of the three kinds of ${ }^{4} \mathrm{He}^{2+}$ ion populations suffering respectively the SDA (red curve), SSA (green curve) and DT (blue curve) mechanisms, and contributing to the total shock front spectrum (represented by a black dashed curve which corresponds to the case $\tilde{V}_{t h i}=10 \tilde{V}_{t h p}$ of Figure 2).

Figures $6 \mathrm{a}-6 \mathrm{~d}$ show the phase space plots of heavy ions at profile A, where the proton population is also shown for reference (Figure 6a). The charge-mass ratios of different species of ions: $\mathrm{H}^{+},{ }^{3} \mathrm{H}^{2+},{ }^{4} \mathrm{He}^{2+}$ or $\mathrm{O}^{8+}$, and $\mathrm{O}^{7+}$ are $1,2 / 3$, $1 / 2$, and $7 / 16$, respectively. The main features are summarized as follows:
[27] 1. In contrast to section 3.1, we found that the diffusion level for the different ions species is almost unchanged (except between protons and helium ions); then, the diffusion mainly depends on the initial thermal velocity (which is the same in present case) as shown in Section 3.1. 
[28] 2. As the ratio $\mathrm{Q} / \mathrm{M}$ decreases (more massive ions), the penetration of heavy ions is deeper and the location of the ions vortex center in the vicinity of shock front shifts downstream.

[29] 3. Figure 6e shows shock front spectra of different heavy ion species. The bump in the high energy range (Figure 2) persists quite well. As the ratio Q/M decreases, the percentage of this monoenergetic ions population (i.e. of reflected ions) decreases as expected, while the power law index $k$ of the middle energy range $(0.01 \sim 0.2)$ increases from 1 to 2.5 .

[30] 4. Figure $6 \mathrm{f}$ shows corresponding downstream spectra of heavy ions. Those spectra follow a power law $\left(\tilde{E}^{-k}\right)$ in the middle energy range $(0.02 \sim 0.4)$, with a $k$ index lower than 0.5 (quite flat). A "shoulder" limit (around $\sim 0.6$ ) is evidenced for each case. The maximum energy cut off $\tilde{E}_{\text {max }}$ is always around 1.5 for all cases (except protons for which $\tilde{E}_{\max }=2.5$ ).

[31] Figures $7 \mathrm{a}-7 \mathrm{~d}$ show the phase space plots of heavy ions at profile B. No reflected ions are evidenced whatever the $\mathrm{Q} / \mathrm{M}$ ratio is. Deeper downstream penetration of heavy ions is observed as the ratio $\mathrm{Q} / \mathrm{M}$ decreases. The shock front spectrum (Figure 7e) presents an unchanged Maxwellianlike shape whatever the ratio is. In contrast, downstream spectra of heavy ions (Figure 7f) show strong changes characterized by a transition from a power law to a Maxwellianlike type as the $\mathrm{Q} / \mathrm{M}$ ratio decreases. The maximum energy cut off is independent on this ratio.

[32] Figures 8a-8d show the phase space plots of heavy ions at profile $\mathrm{C}$ (largest amplitude of the shock front). As the $\mathrm{Q} / \mathrm{M}$ ratio decreases, the upstream excursion of reflected heavy ions (during their gyromotion) increases due to their more massive inertia (similar to Figure 4), and their diffusion slightly increases. However, the vortex location is almost unchanged. Figure 8e shows shock front spectra of heavy ion particles. Low energy range (less than 0.02) of these spectra is unchanged whatever the $\mathrm{Q} / \mathrm{M}$ ratio is. Power law index $k$ in the middle energy range $(0.02 \sim 0.25)$ of these spectra increase with the ratio roughly from 1.6 to 3.3 . The striking feature is in the high energy range $(0.3 \sim 2)$, where a monoenergetic ion population (well-detached Maxwellianlike distribution) is clearly evidenced whatever $\mathrm{Q} / \mathrm{M}$ is. The location of this bump is always centered on roughly the same energy value $(\tilde{E} \sim 0.6)$; its amplitude (i.e. the percentage of reflected ions) and its width (heating) stay almost unchanged. Figure $8 \mathrm{f}$ shows downstream spectra of heavy ions. Low energy range $(0.001 \sim 0.08)$ of these spectra is no monotonic versus $\mathrm{Q} / \mathrm{M}$ ratio. Power law index $k$ in the middle energy range $(0.08 \sim 1.5)$ of these spectra slightly decreases with $Q / M$. Again, a same shoulder value is evidenced in the high energy range $(>1.5)$ for all $\mathrm{Q} / \mathrm{M}$ values, and a similar observation applies for the maximum energy cut off (around 2).

[33] In summary, subsections 3.1 and 3.2 indicate that the fraction of reflected ions increases as their charge-mass ratio decreases and their initial thermal velocity increases. Profile $\mathrm{A}$ and $\mathrm{C}$ reveal to be quite efficient for reflecting and accelerating heavy ions rather than profile $\mathrm{B}$.

\subsection{Particle Trajectory Analysis}

[34] The following question persists: is there any dominant acceleration mechanism responsible for the formation of high energy heavy ions? In particular, what mechanism contributes to the formation of monoenergetic ion population (bump) in shock front spectra as that observed for profile A (Figures 2e and 6e) and profiles $\mathrm{C}$ (Figures $4 \mathrm{e}$ and $8 \mathrm{e}$ ). In order to answer these questions, time trajectory and energization of a few heavy ions have been analyzed from results of profile A chosen as an example (Figure 2d). Results are summarized in Figure 9 where three kinds of heavy ions suffering different energization mechanisms have been identified: the shock drift acceleration (SDA) ions, the shock surfing acceleration (SSA) ions, and the directly transmitted (DT) ions. The identification method for identifying each ion population is analogous to that already applied to protons in a previous paper [Yang et al. 2009a]. Heavy ion trajectories are reported in Figures 9a-9c; Figures 9d-9f show the variations of the corresponding kinetic energy versus X. The upstream edge of the foot, the locations of the ramp and of the overshoot are indicated by red, black and cyan as vertical dashed lines, respectively. Figure 9a shows the trajectories of two typical SDA ${ }^{4} \mathrm{He}^{2+}$ ions in shock rest frame. The black curve is for a standard SDA ion that gains energy during the reflection between the foot (red vertical dashed line) and ramp (black vertical dashed line). The blue curve denotes a crossing SDA ion [Lever et al., 2001] that passes through the overshoot before re-entering upstream during its gyromotion. Figure 9d shows that energization of SDA-crossing is much higher than that of standard SDA, and this energization takes place mainly behind the overshoot and not within the shock front (including the ramp and the foot). Figure $9 \mathrm{~b}$ shows the trajectory of a SSA ${ }^{4} \mathrm{He}^{2+}$ ion which is primarily reflected by the local longitudinal electric field instead of doing a large gyromotion within the shock front. This particle only suffers one bounce because the cross-shock potential is not strong enough to produce a multi-reflected ion (MRI). Figure 9e shows that this SSA ion (one bounce) has poor energization as compared to the SDA ion (Figure 9d). Figure 9c shows two typical DT ${ }^{4} \mathrm{He}^{2+}$ ions trajectories. The "black" ion corresponds to a directly transmitted ion without any energy enhancement (Figure 9f), while the "blue" ion describes a gyro-motion immediately downstream of the overshoot and gains energy from the transverse electric field (in the accelerating phase of downstream drift motion). This acceleration mechanism was proposed by Toida and Ohsawa [1997] and will be discussed in section 5. This selection in SSA, SDA and DT ions has been applied to the shock front spectrum of Figure 2d. Statistical results are reported in Figure 9g, and indicate that SDA (red) helium ions are the most energetic ions and are mainly responsible for the bump in the high energy range (monoenergetic population). In contrast, the SSA (green) helium ions succeed to reach a part of the high energy range (their average energy is about 0.5 ), but contribute only a little to the bump (percentage much less than that of SDA ions). At least, most DT ions contribute to the low energy part of spectrum; only a few succeed to reach an energy comparable to that of the SSA ions. Extensively, complementary statistics have been performed for different charge-mass ratios (not shown here) and have clearly evidenced that the high energy part of the shock front energy spectrum (characterized by the bump) corresponds always to SDA ions; SSA ions only bring a limited contribution. One invoked reason is the broad thickness of the ramp. Indeed, a 
very thin ramp is required for the SSA mechanism to be efficient [Zank et al., 1996].

\section{Simulation Results: Time-Evolving Shock Profiles}

[35] We investigate particle acceleration when the shock is continuously nonstationary as shown in Figure 1 (left). The period of self-reformation cycle is about $\tilde{t}_{S R}=256$. Test particles are initially distributed evenly (50 particles per cell) within a wide upstream region at a chosen starting time $\tilde{t}=$ 628 , and have enough time (several reformation cycles) to interact with the propagating shock. In order to compare with the fixed shock profiles results (Section 3), we separate this section into two parts dedicated respectively to the impact of the initial thermal velocity and of the charge-mass ratio. In each part, we will consider three different ending times. First, let us consider the time $\tilde{t}=1552$ for instance. We leave the heavy ions interacting with the nonstationary shock between $628<\tilde{x}<1552$; then, these continuously suffer the fields fluctuations at the shock front and within the downstream region during this time interval. This is in contrast with results of Section 3 where ions interacted with selected profiles fixed within the whole spatial range (whole shock front and downstream region). Presently, the downstream spectrum obtained at time $\tilde{t}=1552$, is calculated between the overshoot location of the current shock profile (at $\tilde{t}=1552$ ) and the overshoot location of the analogous profile obtained one reformation cycle before (at $\tilde{t}=1284$ ). In other words, the downstream spectrum is space (and time) integrated within the locations of the "old" and the "new" overshoots. This allows to compare with results obtained at same time $\tilde{t}=1552$ with the fixed profile A of Figure $2 \mathrm{f}$. At the same time $\tilde{t}=1552$, the shock front spectrum is calculated from the upstream edge of the foot defined by $\tilde{B}_{z}>$ $\tilde{B}_{o} 0.1$ (where 0.1 is slightly higher than the maximum amplitude of upstream B field turbulence) to the ramp location (defined by the maximum peak of $\tilde{E}_{x}$ ). Similar comparison can be performed for shock front spectrum of profile A of section 3 (Figure 2e). Same procedure will be used with times $\tilde{t}=1600$ and $\tilde{t}=1744$ in order to compare with results obtained for profiles $\mathrm{B}$ (Figure $3 \mathrm{e}$ ) and $\mathrm{C}$ (Figure 4e) for the shock front spectra and for the downstream spectra (Figures $3 \mathrm{f}$ and $4 \mathrm{f}$ ). In all cases, the box size is identical to that used for results of Section 3.

\subsection{Impact of Initial Thermal Energy}

[36] First, let us analyze the impact of different initial thermal velocities on heavy ions interacting with a timeevolving shock. At $\tilde{t}=1552$, heavy ions have interacted with the self-reforming shock over a long time range $\Delta \tilde{t}=$ $924 \approx 3.6 \tilde{t}_{S R}$. The phase space plots of helium ions are shown in Figures 10a-10d. Those ions have an initial Maxwellian distribution with different thermal velocity equal to $0.5 \tilde{V}_{t h p}$ (Figure 10a), $\tilde{V}_{t h p}$ (Figure 10b), $5 \tilde{V}_{t h p}$ (Figure 10c), and $10 \tilde{V}_{\text {thp }}$ (Figure 10d). Magnetic (blue curve) and longitudinal electric (red curve) fields are also plotted for reference. Comparison between Figures 2 and 10 evidence the following points:

[37] 1. Figures $10 \mathrm{a}$ and $10 \mathrm{~b}$ show that most heavy ions succeed to pass downstream with strongly distorted trajectories due to the succession of coherent structures as these interact with the varying shock front (vortices). The reminiscent tracks of these coherent structures still persist at that time in phase space and extend within a larger downstream domain.

[38] 2. Figures $10 \mathrm{c}$ and $10 \mathrm{~d}$ show an ion diffusion larger than that in Figure 2. Moreover, the location of the vortex center in phase space is almost the same as that in Figure 2.

[39] 3. Figures 10e and 10f show the shock front spectra and the downstream spectra at $\tilde{t}=1552$, respectively. Shock front spectra are measured from the upstream edge of the foot $(X=5419)$ to the ramp position $(\tilde{X}=5359)$. The downstream spectra are measured between the overshoot location $\tilde{X}=5353$ and the previous similar overshoot located at $\tilde{X}=5126$ (defined at $\tilde{t}=1284$ ). Shock front spectra (Figure 10e) do not show noticeable differences except a higher percentage of reflected ions with respect to Figure 2. In addition, Figure 10f shows again a power law form $\left(\tilde{E}^{-k}\right)$ in the middle energy range $(0.01$ to 0.8$)$, but the index $k(\sim 0.75)$ stays almost unchanged whatever $\tilde{V}_{t h i}$ is.

[40] At $\tilde{t}=1600$, heavy ions have interacted with the selfreformed shock over a longer time range $\Delta \tilde{t}=972 \approx 3.8 \tilde{t}_{S R}$. Corresponding phase space plots (Figures 11a-11d) do not show any noticeable difference with those of Figure 10 except larger ion diffusion. The fraction of reflected heavy ions is substantially more than that in Figure 3. Figures $10 \mathrm{e}$ and 10f show the shock front spectra and the downstream spectra at $\tilde{t}=1600$, respectively. Shock front spectra are measured from the upstream edge of the foot $(\tilde{X}=5465)$ to the ramp position $(\tilde{X}=5436)$. The downstream spectra are measured between the overshoot location $\tilde{X}=5367$ and the previous similar overshoot located at $\tilde{X}=5150$ (defined at $\tilde{t}$ $=1320$ ). In contrast with Figure 3, Figure 10e shows a clear bump for high thermal velocity cases. The amplitude of this bump is strongly enhanced for high $\tilde{V}_{t h i}$. Figure $10 \mathrm{f}$ shows a clear power law (instead of Maxwellian) with an index varying from 0.85 (Figure 10a) to 0.57 (Figure 10d) within the middle energy range ( 0.02 to 0.6$)$.

[41] At $\tilde{t}=1744$, heavy ions have interacted with the selfreformed shock over a longer time $\tilde{t}=1116 \approx 4.36 \tilde{t}_{S R}$. Corresponding phase space plots (Figures 12a-12d) are similar to those of Figures 10 and 11, but strongly differ from the stationary shock profile (Figure 4). Figures $12 \mathrm{e}$ and $12 \mathrm{f}$ show the shock front spectra and the downstream spectra at $\tilde{t}=1744$, respectively. Shock front spectra are measured from the upstream edge of the foot $(\tilde{X}=5563)$ to the ramp position $(\tilde{X}=5537)$. The percentage of reflected heavy ions (bump amplitude in high energy range in Figure 12e) is less than that of Figure 4. For low initial $\tilde{V}_{t h i}\left(<\tilde{V}_{t h p}\right)$, reflected heavy ions are totally absent. The reason is due to the fact that the electromagnetic fields at the shock front increase more rapidly (as driven by reflected protons in the PIC simulation) as compared to the time during which heavy ions will interact with such high amplitude front. In other words, the shock does not maintain a high amplitude front during a time long enough to reflect a noticeable number of new incoming heavy ions. Herein, inertia effects of heavy ions dominate. For high initial $\tilde{V}_{t h i}\left(>\tilde{V}_{t h p}\right)$, the heavy ions suffer some reflection even within a full self-reformation cycle. The downstream spectra are measured between the overshoot $(\tilde{X}=5528)$ and the previous similar overshoot located at $\tilde{X}=5306$ (defined at $\tilde{t}=1488$ ). Figure $12 \mathrm{f}$ shows big differences with Figure 4 due to the absence of large gyrating 

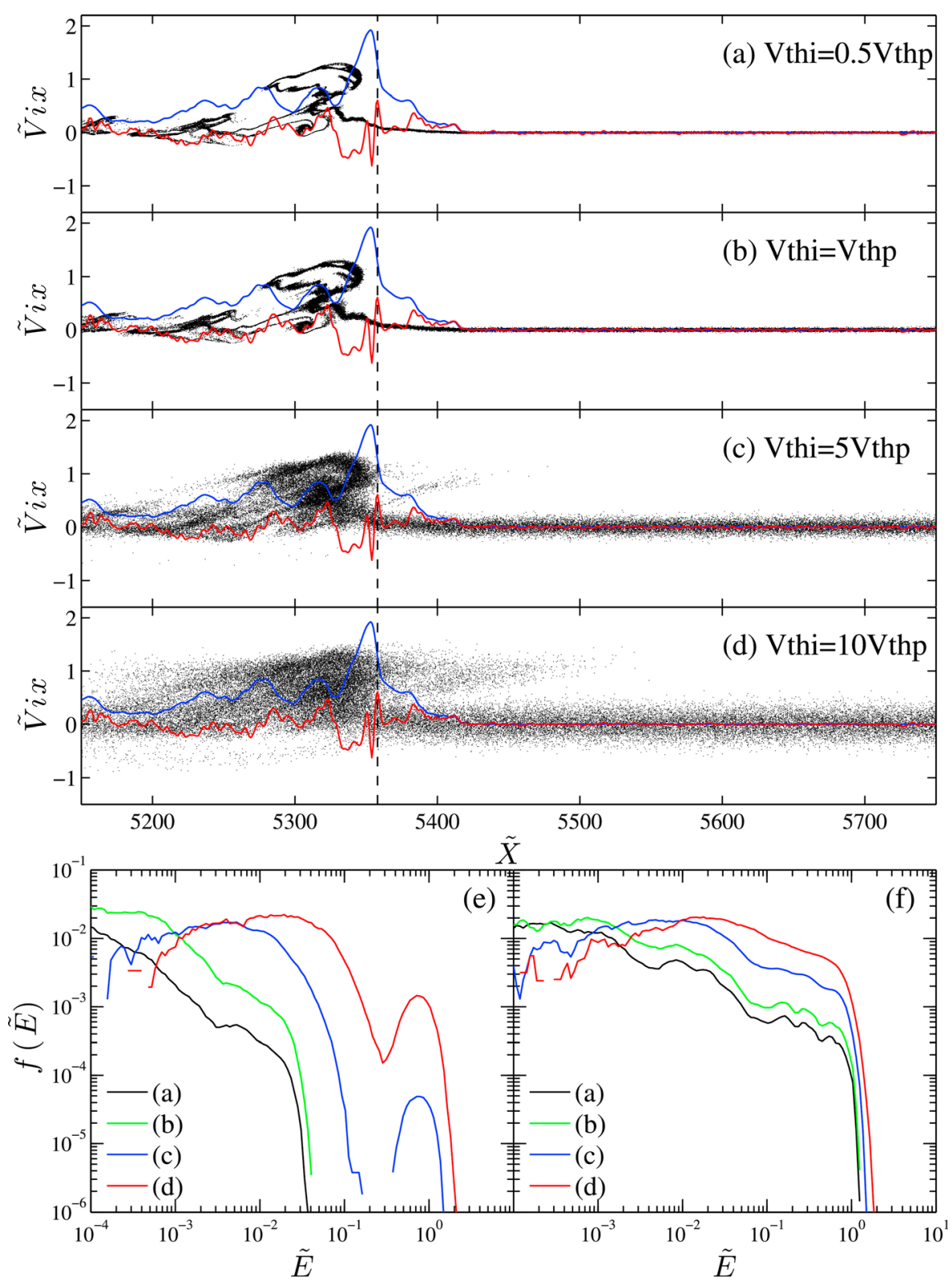

Figure 10. (a-d) The phase space plots $\left(\tilde{V}_{i x}\right.$ versus $\left.\tilde{X}\right)$ of heavy ion ${ }^{4} \mathrm{He}^{2+}$ with different initial thermal velocities for a continuously time self-reforming shock between times $\tilde{t}=628$ and $\tilde{t}=1552$. Main magnetic field $\tilde{B}_{z}$ (blue) and electrostatic field $\tilde{E}_{x}$ (red) are also shown at time $\tilde{t}=1552$ for reference in each plot. (e and f) The corresponding normalized energy spectra calculated within the shock front and downstream regions, obtained from Figures 10a-10d; each case (Figures 10a-10d) of Figures 10e and 10f is identified by different colors.

heavy ions in the downstream region; instead the heavy ions are phase mixed downstream by the self-reformation of the shock front.

[42] In summary, the nonstationary effects smooth out the strong variation evidenced in the percentage of reflected ions (from $0 \%$ to $100 \%$ ) for stationary profiles. Instead, these contribute to maintain a noticeable reflection characterized by the formation of a monoenergetic population (bump) in the high energy range of the shock front spectra. Moreover, an additional filtering effect due the initial thermal velocity is observed in the sense that ion reflection is only observed for high $\tilde{V}_{t h i}\left(>\tilde{V}_{t h p}\right)$. At least, the nonstationary effects lead to a power law in all downstream spectra due to the ions phase mixing fed by the shock self reformation; these effects dominate those due to the initial thermal velocity variation. The index of this power law varies only slightly with $\tilde{V}_{t h i}$.

\subsection{Impact of Charge-Mass Ratio}

[43] All heavy ions species have now an initial Maxwellian velocity distribution with the same thermal velocity $\tilde{V}_{t h i}=$ $5 \tilde{V}_{t h p}$ (the subscript "i" holds for different species of ions). 

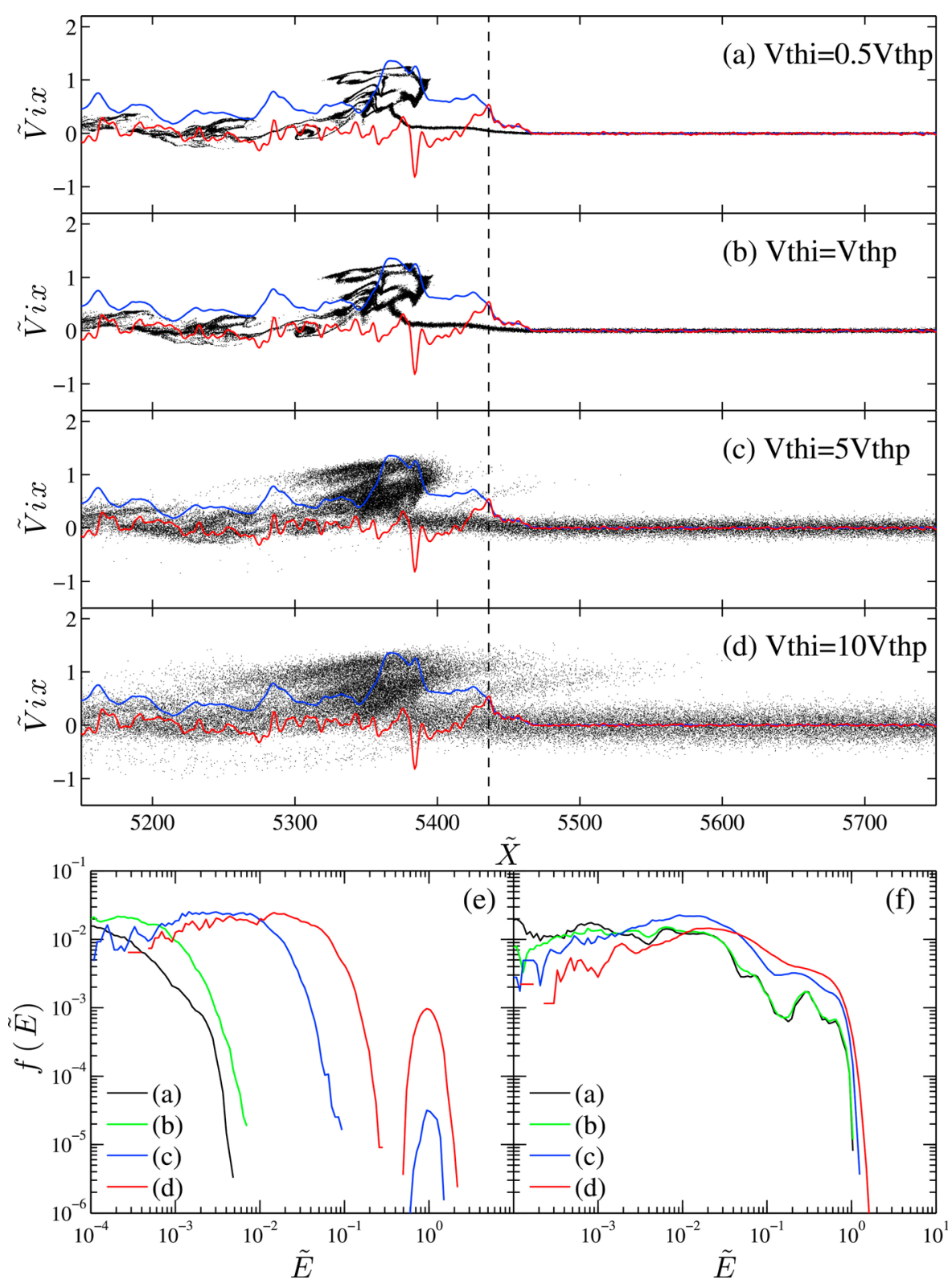

Figure 11. (a-f) Similar plots as Figure 10 for a continuously time self-reforming shock and measured at $\tilde{t}=1600$. Field components are also shown for reference in Figures $11 \mathrm{a}-11 \mathrm{~d}$.

The other setups of heavy ion test particles and spectrum calculation boxes are the same as those used in section 4.1. Figures 13a-13d shows phase space plots with different charge-mass ratios at $\tilde{t}=1552$. By this time, heavy ions have interacted with this self-reformed shock over a long time $\tilde{t}=924 \approx 3.6 \tilde{t}_{S R}$, which roughly covers $0.875,0.58,0.44$ and $0.38 \tilde{\tau}_{c i}$, where $\tilde{\tau}_{c i}$ is the upstream gyroperiod of $\mathrm{H}^{+}$, ${ }^{3} \mathrm{H}^{2+},{ }^{4} \mathrm{He}^{2+}$ or $\mathrm{O}^{8+}$, and $\mathrm{O}^{7+}$ ions respectively. For this continuously time-evolving shock, all species of ions have a high percentage of reflected ions. Due to the inertia effects, the gyration of heavy ions has just started downstream in the overshoot. Figure 13e shows that the shock front spectra are similar to the results of Figure 6e (fixed stationary shock) except that the bump in the high energy range (due to reflected ions) is better separated from the core (low energy part) of the spectra. The percentage of reflected heavy ions (bump) is decreasing with the $\mathrm{Q} / \mathrm{M}$ ratio. However, this percentage is higher than for a stationary shock (Figure 6e). Figure $13 \mathrm{f}$ shows no noticeable change with Figure 6f, except that a power law is evidenced only for low $\mathrm{Q} / \mathrm{M}$ ratio $\left({ }^{4} \mathrm{He}^{2+}\right.$ or $\mathrm{O}^{8+}$, and $\left.\mathrm{O}^{7+}\right)$. Let us note that similar simulations have been performed for two additional cases: (1) until $\tilde{t}=1600$ where heavy ions have interacted with the shock over a time range $\tilde{t}=972 \approx 3.8 \tilde{t}_{S R}$, which roughly equals to $0.92,0.614,0.46$ and $0.4 \tilde{\tau}_{c i}$ (upstream gyroperiod) for $\mathrm{H}^{+},{ }^{3} \mathrm{H}^{2+},{ }^{4} \mathrm{He}^{2+}$ or $\mathrm{O}^{8+}$, and $\mathrm{O}^{7+}$ ions, respectively; (2) until $\tilde{t}=1744$, where heavy ions have interacted with the shock for a time range $\tilde{t}=1116 \approx$ $4.36 \tilde{t}_{S R}$, which roughly equals to $1.06,0.705,0.53$ and $0.46 \tilde{t}_{S R}$ (upstream gyroperiod) for $\mathrm{H}^{+},{ }^{3} \mathrm{H}^{2+},{ }^{4} \mathrm{He}^{2+}$ or $\mathrm{O}^{8+}$, 

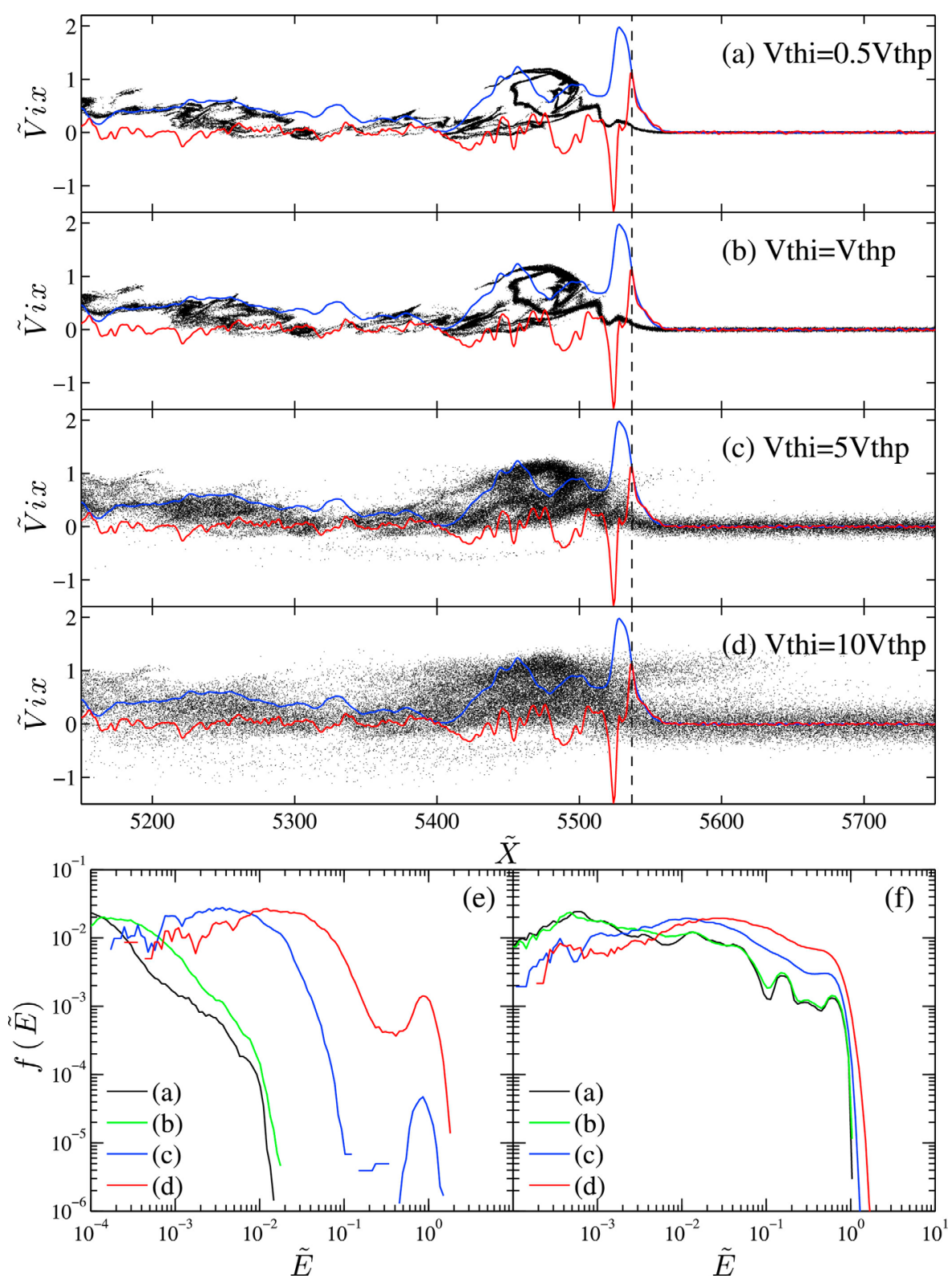

Figure 12. (a-f) Similar plots as Figure 10 for a continuously time self-reforming shock and measured at $\tilde{t}=1744$. Field components are also shown for reference in Figures 12a-12d.

and $\mathrm{O}^{7+}$ ions, respectively. These both last cases are not shown here, since no noticeable difference have been evidenced with respect to results of Figure 13.

[44] In summary, the nonstationary effects contribute to the formation of reflected ions (and reinforce the amplitude of the corresponding bump) in the shock front spectra. However, these effects have a weaker impact in the downstream spectra for high $\mathrm{Q} / \mathrm{M}$ ratio cases

\section{Discussion and Comparison}

[45] This section contains two parts: one dealing with comparison with previous simulations and associated theoretical models, the other with the application to solar energetic particle events.

\subsection{Comparison With Previous Simulations and Theoretical Models}

[46] In this subsection, we compare our present results with those of previous papers. First, Toida and Ohsawa [1995, 1997] have investigated the heavy ion acceleration at a stationary perpendicular shock by using both analytical approach and PIC simulations including four different ions species $(\mathrm{H}, \mathrm{He}, \mathrm{O}, \mathrm{Fe})$. The authors found the following results: (1) heavy ions are accelerated along the direction parallel to the wave front under the transverse electric field $\left(\tilde{E}_{t y}\right)$, and light ions (protons) are accelerated through reflection by the longitudinal electric field; (2) the acceleration of protons is very rapid while that of heavy ions is quite slow (it is even slower as the charge to mass ratio decreases); (3) all heavy ions are accelerated (by $\tilde{E}_{t y}$ field) 

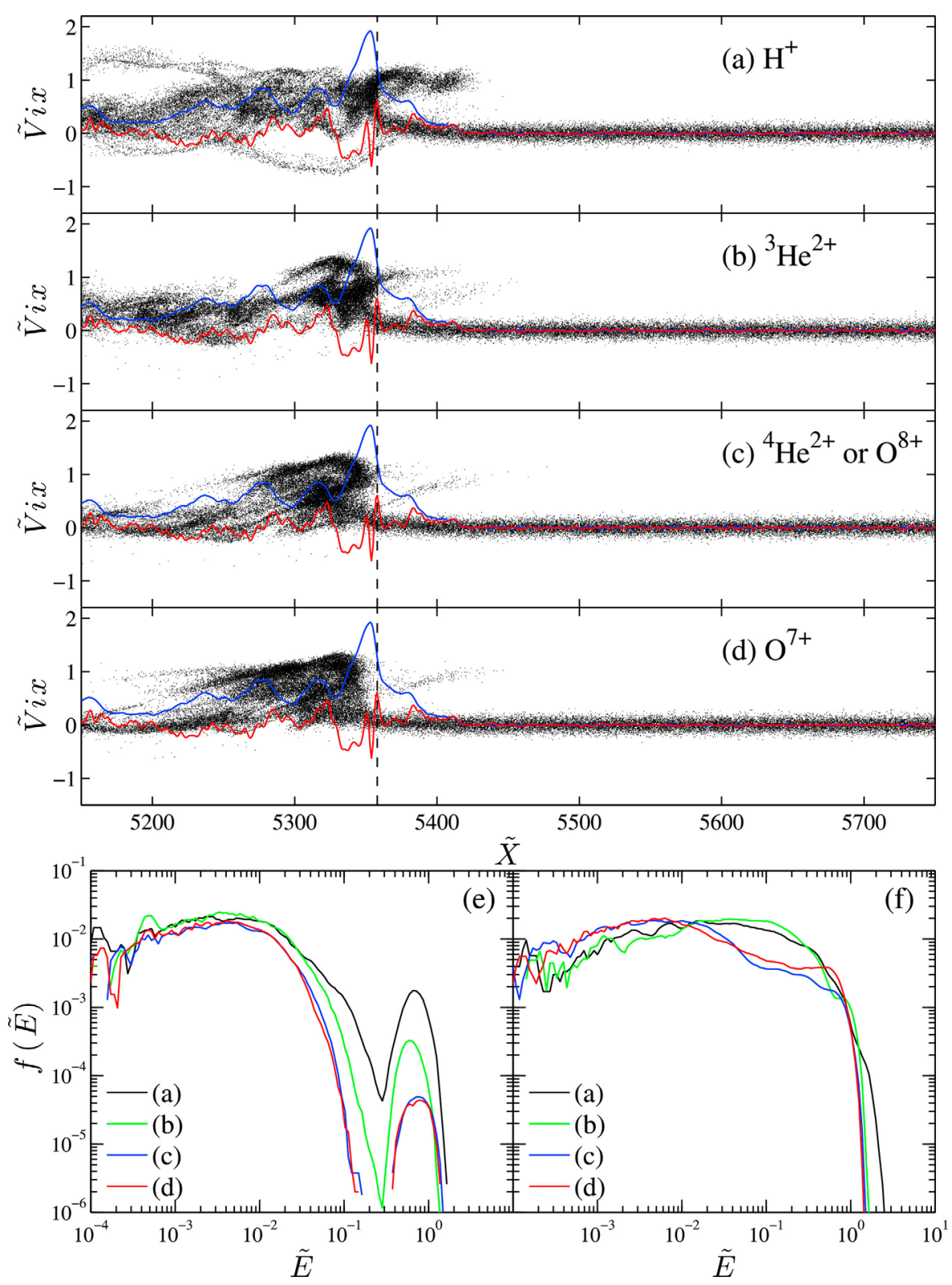

Figure 13. (a-d) The phase space plots $\left(\tilde{V}_{i x}\right.$ versus $\left.\tilde{X}\right)$ of different ion species with the same initial thermal speed $\left(\tilde{V}_{t h i}=5 V_{t h p}\right)$ for a continuously time self-reforming shock measured at $\tilde{t}=1552$. Main magnetic field $\widetilde{B}_{z}$ (blue) and electrostatic field $\widetilde{E}_{x}$ (red) are also shown at time $\tilde{t}=1552$ for reference in each plot. (e and f) The corresponding normalized energy spectra calculated within the shock front and downstream region as obtained from Figures 13a-13d; each case (Figures 13a-13d) of Figures 13e and $13 \mathrm{f}$ is identified by different colors.

while only a part of incoming protons is accelerated by reflection; and (4) accelerated heavy ions reach a maximum $\mathrm{y}$-velocity which is independent of the charge to mass ratio and can be written as $V_{T O}=\frac{B_{\max }-B_{o}}{B_{\max }+B_{o}} M \nu_{h}$ [Toida and Ohsawa, 1997], where $\nu_{h}$ is the propagation speed of the wave. However, two important questions are still unanswered: (1) Why are some heavy ions reflected in our present simulations? (2) What is the impact of the shock front nonstationarity on the maximum velocity $\tilde{V}_{T O}$ of heavy ions?

[47] We have completed our analysis with additional simulations in order to address these questions. Figure 14 shows the percentage of reflected heavy ions obtained for one stationary shock versus their initial thermal velocity along $x$ direction; herein, we take profile $\mathrm{A}$, which includes typical foot, ramp and overshoot structures within the shock transition region. The charge-mass ratios of heavy ions: ${ }^{3} \mathrm{H}^{2+},{ }^{4} \mathrm{He}^{2+}$ or $\mathrm{O}^{8+}$, and $\mathrm{O}^{7+}$ are $2 / 3,1 / 2$, and $7 / 16$ respectively. Figure 14a shows that this percentage increases with the charge-mass ratio and the initial thermal velocity. It evidences clearly that as the initial thermal velocity is below a certain threshold (which also increases with the chargemass ratio), no ions are reflected (percentage is around zero). The initial conditions used by Toida and Ohsawa are indicated by a vertical arrow. Our results recover those 

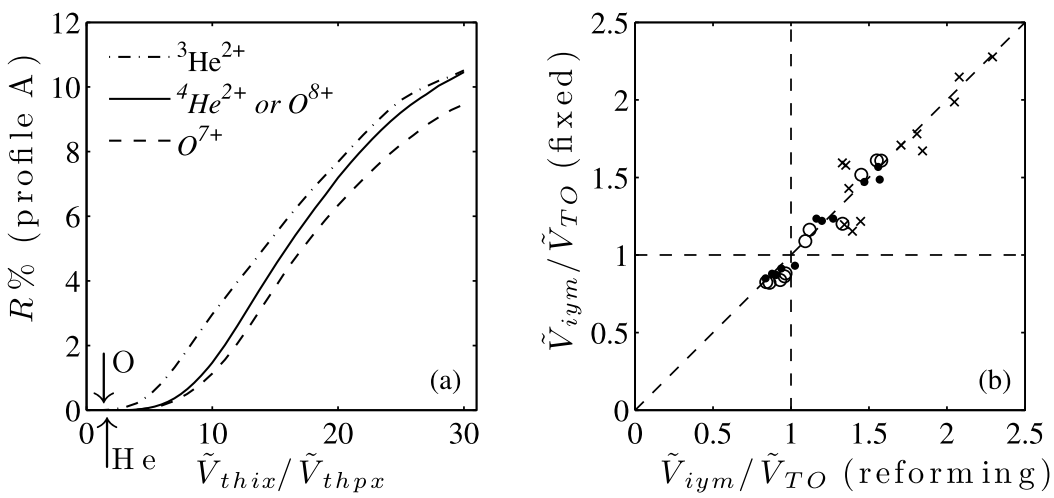

Figure 14. (a) The percentages of reflected heavy ions at shock profile $A$ versus their initial thermal $\mathrm{x}$-velocity component normalized to the proton thermal $\mathrm{x}$-velocity. Different heavy ion species ${ }^{3} \mathrm{H}^{2+}$, ${ }^{4} \mathrm{He}^{2+}$ or $\mathrm{O}^{8+}$, and $\mathrm{O}^{7+}$ are denoted by dash-dotted, solid and dashed curves, respectively. The downward arrow (near the origin) corresponds to the conditions used by Toida and Ohsawa [1997]. (b) The ratio $\tilde{V}_{i y m} / \tilde{V}_{T O}$ calculated for fixed shock cases versus those obtained for continuously time-evolving (reforming) shock. $\tilde{V}_{i y m}$ denotes the maximum heavy ion y-velocity measured around the shock front, while $\tilde{V}_{T O}$ indicates the value deduced from the model of Toida and Ohsawa [1997]. Results are represented by dots ("•"), circles ("○") and crosses ("×"), and measured respectively for different fixed shock profiles A, B and C (vertical axis) and measured at the same times $\tilde{t}=1552,1600$, and 1744 in the continuously time self-reforming shock (horizontal axis).

of Toida and Ohsawa [1995, 1997] in low initial thermal velocity cases, where no heavy ions are reflected.

[48] In the present analysis, we found that the percentage of reflected heavy ions not only depends on the charge-mass ratio and initial thermal velocity but also depends on the shock front structure. Indeed, for profile B (Figures 3 and 7), the density of reflected ions decreases because of the weak longitudinal electric field amplitude and broad shock ramp, while for profile $\mathrm{C}$ (Figures 4 and 8 ), this fraction is strongly enhanced due to the steep shock ramp and high cross-shock potential amplitude which is dominated by Hall effect [Yang et al., 2009b].

[49] In addition, Figure $14 \mathrm{~b}$ shows the ratios $\tilde{V}_{i y m} / \tilde{V}_{T O}$ measured for the three fixed shock profiles $\mathrm{A}, \mathrm{B}$ and $\mathrm{C}$ (stationary cases), and at three same corresponding times $\tilde{t}=$ 1552, 1600 and 1744 for time-evolving shock profiles (nonstationary case); $\tilde{V}_{i y m}$ indicates the maximum y-velocity of heavy ions around the shock front, and $\tilde{V}_{T O}$ denotes the expected value obtained from the theory of Toida and Ohsawa [1997]. The crossing point of the dashed lines corresponds to an "ideal" agreement with the theoretical model. Our results show that two-thirds of the numerical values $\tilde{V}_{i y m}$ obtained in the different cases of our simulations (stationary and nonstationary cases) are much higher than the theoretical value (i.e. are above this crossing point). This is particularly true for shock profile $\mathrm{C}$ at $\tilde{t}=1744$ denoted by crosses " $\mathrm{x}$ " which are spreading far from the crossing point, and where most incident heavy ions can be reflected and accelerated by SDA/SSA mechanisms. For those heavy ions, as $\tilde{V}_{i y}$ increases during their reflection, $\tilde{V}_{i x}$ increases rapidly which is neglected in the Toida and Ohsawa's model.

[50] Secondly, we compare our results with those obtained by Chapman et al. [2005] who have investigated the impact of helium percentage in the upstream region on downstream protons and helium spectra. Their main results may be summarized as follows: (1) the self-reformation of the shock front (initially driven by the accumulation of reflected protons) changes noticeably as the relative density of helium ions (versus protons) increases above $\sim 10 \%$ in the upstream region; (2) these changes in the shock front are accompanied by modulated downstream spectra of both protons and helium ions. Herein, we investigate the effect of nonstationary shock front on downstream particle spectra. According to Chapman et al.'s results, our results can be applied to the low helium percentage $(<10 \%)$ cases.

[51] It is worth mentioning that the heavy ion spectra measured within the shock front and the downstream region (Figures $10 \mathrm{e}, 10 \mathrm{f}, 13 \mathrm{e}$, and 13f) are also highly modulated by the nonstationarity of the shock front. In order to compare more accurately with the previous work of Chapman et al. [2005], we calculate the downstream energy spectra of protons and helium ${ }^{4} \mathrm{H}^{2+}$ particles at different times within a reformation cycle in the downstream rest frame. Simulation boxes used for calculating present spectra are identical to those mentioned in section 4; moreover, the energy values are normalized to the particle injection energy " $\tilde{E}_{i n j}$ ", which is defined by Chapman et al. [2005]. The initial proton thermal velocity $\tilde{V}_{t h p}$ is also close to that used by Chapman et al. [2005]; herein we use $\tilde{V}_{t h i} / \tilde{V}_{t h p}=0.7$ for helium ions. Figure 15 shows that proton spectra have a Maxwellian shape (almost straight line in semilog scale plots) while helium spectra have power law shapes. The shapes and the variations of the downstream spectra for both populations are similar respectively to those obtained by Chapman et al. [2005]. In addition, our results evidence that protons (helium) downstream spectra are relatively weakly (strongly) affected by the shock front self-reformation. One striking feature is that the impact of the shock front nonstationarity (within one self-reformation cycle) on energy spectra is comparable to that due to the presence of helium ions (in particular above 10\%) mentioned by Chapman et al. [2005] and integrated over several self-reformation cycles. 

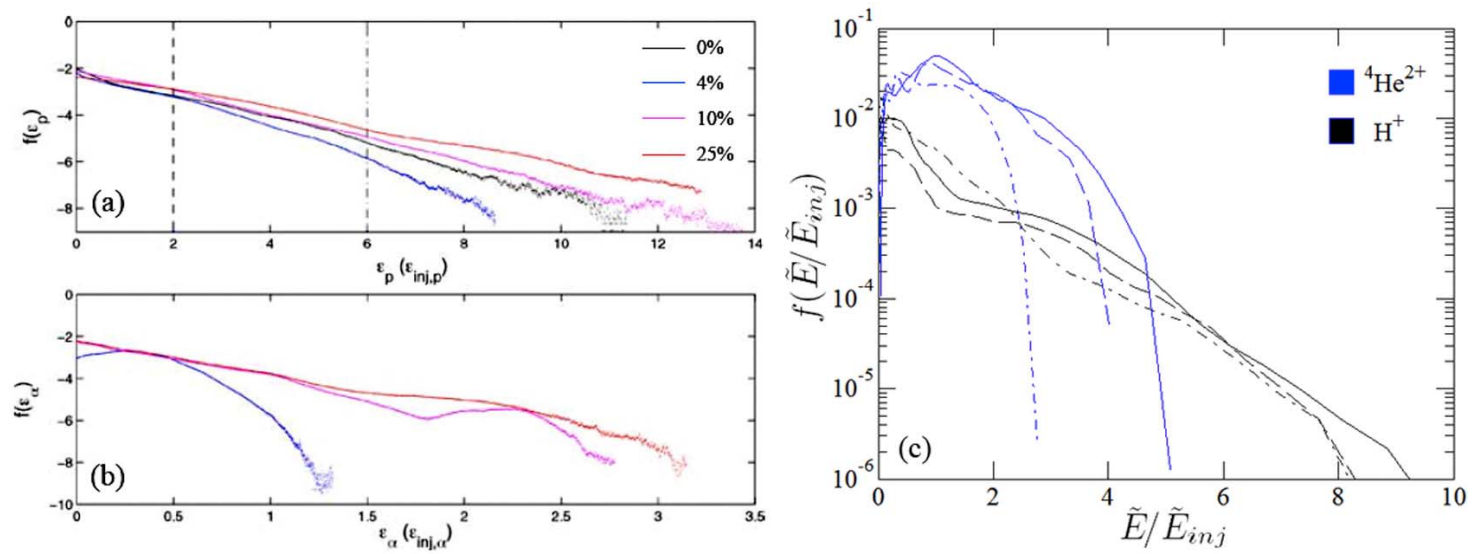

Figure 15. (a and b) Downstream energy spectra of $\mathrm{H}^{+}$(subscript "p") and ${ }^{4} \mathrm{He}^{2+}$ (subscript " $\alpha$ ") ions obtained by Chapman et al. [2005], respectively. (c) Corresponding downstream energy spectra of $\mathrm{H}^{+}$ (black) and ${ }^{4} \mathrm{He}^{2+}$ (blue) ions measured in our case at different times $(\tilde{t}=1552$ in solid, $\tilde{t}=1600$ in dashed, and $\tilde{t}=1744$ in dashed-dotted curves) for a continuously time-evolving shock. Those spectra are measured in the downstream frame and normalized to the upstream energy $\tilde{E}_{i n j}$ as given by Chapman et al. [2005].

\subsection{Comparison With Experimental Observations and Models of Solar Energetic Particle (SEP) Events}

[52] It is worth reviewing shortly the features of heavy ion energy spectra in large gradual SEP events. Here, we only concentrate on the observational/experimental energy spectra of ions in a series of gradual SEP events associated with CME-driven shocks. Reames et al. [1997] reported energy spectra of ions from a gradual event observed by WIND spacecraft (1995 October 20th). The slope value of energy spectra (averaged over different events) measured for different ion species $(\mathrm{H}, \mathrm{He}, \mathrm{C}$ etc.) ranged roughly from 1 to 1.3 within the energy range 0.0002 1. Tylka and Lee [2006] studied two large gradual SEP events respectively on 2002 April 21 and 2002 August 24, which have similar fast CMEs and flares origins. The energy spectra of different ions species observed by ACE and WIND spacecrafts have been fitted to some familiar empirical power-law distributions [Ellison and Ramaty, 1985; Band et al., 1993]. The corresponding slope values deduced for the two 2002 events ranged roughly from 0.8 to 1.5 within the energy range $0.0001 \sim 0.01$, and from 2.4 to 4.2 within the range $0.01 \sim 5$, respectively. Those spectra have been measured from upstream to downstream of the CME-driven shocks over 23 days. Moreover, the authors found that these slope values observed in gradual SEP events not only depend on the shock acceleration mechanism and the transport through the proton-generated Alfven turbulence upstream of the shock [Ng et al., 2003], but also highly depend on the location of the satellite with respect to the curved shock front (as shown by Reames [1999, Figure 3.4]).

[53] In order to interpret experimental data, a large amount of SEP models have been processed in order to analyze the particle acceleration and transport in SEP events associated with CME-driven shocks. Zank et al. [2000] have used a one-dimensional hydrodynamic code together with diffusive shock acceleration theory in order to model the evolution of a CME-driven shock and to produce the accelerated particle spectrum. Their model is only applicable to extremely strong shocks, due to the use of the Bohm form of the spatial diffusive coefficient [Berezhko and Völk, 2007; Shalchi, 2009]. The model presented by Rice et al. [2003] is an extension of Zank et al. [2000] in that they calculated the intensity of the upstream Alfvén waves driven by the accelerated particles. This allows the spatial diffusion coefficient to be calculated self-consistently, using the steady-state solution of Gordon et al. [1999], rather than invoking the Bohm limit. They have extended the acceleration model of Zank et al. [2000] to shock waves of arbitrary strength. Their main results can be summarized as follows: (1) both the energy spectra upstream and downstream of the shocks exhibit a power-law distribution $\left(\tilde{E}^{-k}\right)$; (2) the slope values of these spectra decrease with the increasing shock compression ratio $\left(B_{D S} / B_{U S}\right.$, where $B_{D S}$ and $B_{U S}$ are respectively the downstream and upstream magnetic field of a perpendicular shock), and roughly range from 1 to 2.5 .

[54] A three-dimensional SEP model has been also performed by using similar method [Lee, 1983; Lee and Ryan, 1986]. The authors modeled the shock as a spherically symmetric, infinite-strength, self-similar, stationary blast wave and made some strong assumptions (e.g., very high blast wave velocity such as $V_{\text {shock }}=1000 \mathrm{Km} / \mathrm{s}$, to make their model tractable). The slope values of their power -law spectra are within a wide range which can be easily consistent with observations. However, shocks driven by fast CME's are generally believed to be the dominant accelerators in large gradual SEP events. A key challenge supporting this scenario has been the highly variable spectral and compositional characteristics of these events above a few tens of $\mathrm{MeV}$ per nucleon [Tylka et al., 2005]. In order to resolve this issue, Tylka and Lee [2006] have developed an analytical model in which they proposed that this variability results from the interplay of two factors: (1) the variation of the shock-normal angle $\theta_{B n}$ as the shock moves outward from the Sun, and (2) a compound seed population, typically comprising at least suprathermal particles from the corona (or solar wind) such as ${ }^{4} \mathrm{He}^{2+}, \mathrm{O}^{6+}$ and $\mathrm{Fe}^{10+}$ ) and suprathermal 
particles from flares (such as ${ }^{3} \mathrm{He}^{2+}, \mathrm{O}^{8+}$ and $\mathrm{Fe}^{20+}$ ). The functional form suggested by Ellison and Ramaty [1985, equation 1] and Tilka and Lee [2006; equation 1] is used to describe the spectrum of ions accelerated by diffusive shock acceleration. They found that the fitted power law indices for oxygen and iron spectra at quasi-perpendicular shock can reach values around 2.94 and 2.54 , respectively.

[55] Sandroos and Vainio [2007] retrieved the results of previous analytical models [Tylka and Lee, 2006] by using Monte Carlo simulations. In their simulations, the initial spectra of all ion species follow a power-law distribution, and the shock is modeled as a spherical discontinuity having a constant velocity, gas compression and expanding towards all directions as a bubble. But no information is provided about the shock front thickness and nonstationary effects. The other initial parameters, e.g., ions charge-mass ratios correspond to the values inferred by Tylka and Lee [2006]. They found that their model is in qualitative agreement with both the model of Tylka and Lee [2006] and observations.

[56] However, diffusive shock acceleration does not work well at quasi-perpendicular shocks $\left(\theta_{B n}>45^{\circ}\right)$, where the reflected ions return to the shock front almost immediately due to their gyro-motion in the upstream magnetic field. Decker and Vlahos [1986] presented a study of shock acceleration by integrating numerically the test-particle trajectories using magnetic field turbulence that was constructed by superimposing a random component on the background shocked field profile. Decker [1983] investigated the effect of the long wavelength fluctuations on particle acceleration in one-dimensional shock fields. All these above shocks did not include cross-field diffusion. Therefore, Giacalone and Jokipii [1996] have improved the model which is fully three-dimensional so that cross-field diffusion, which is the most relevant form of diffusion at perpendicular shocks, is possible. The slope values of downstream energy spectra roughly range from 1 to 2.3 and depend on the fluctuations scales. Unfortunately, the shock microstructures (such as the magnetic field overshoot, the cross-shock potential and the foot) as those observed during a time-varying self-reforming shock are not included in their model.

[57] In this paper, we compare our results with previous observations [Tylka et al., 2005] and simulations [Tylka and Lee, 2006; Sandroos and Vainio, 2007] obtained on Fe/O ratio spectrum. The main results of these previous works may be summarized as follows: (1) above a few tens of Mev per nucleon, large, gradual solar energetic particle (SEP) events are highly variable in their spectral characteristics (e.g. the abundance ratio of $\mathrm{Fe} / \mathrm{O}$ versus energy) and in elements composition (e.g. the average charge state of Fe versus energy); (2) it is generally believed that this variability results from two factors: shock angle $\theta_{B n}$ (the angle between the shock normal and upstream magnetic field) and a compound seed population (at least from corona and flares); (3) the high energy part of $\mathrm{Fe} / \mathrm{O}$ ratio spectra increases with shock angle and relative percentage of flare seed population. However, some important questions still persist. If one takes a careful look at the characteristic spectra shown in Figures 16a and 16b [from Tylka et al., 2005] several relevant features can be noticed: (1) events with high percentages of $\mathrm{Fe} / \mathrm{O}$ are preferentially associated with large percentage of ${ }^{3} \mathrm{He}$ and large $\theta_{B n}$ values. In con- trast, events with falling $\mathrm{Fe} / \mathrm{O}$ generally have smaller percentage of ${ }^{3} \mathrm{He}$ and are associated with a full range of $\theta_{B n}$ values; (2) moreover, the distribution of colors in the Figure $16 \mathrm{~b}$ hints that among events with falling $\mathrm{Fe} / \mathrm{O}, \mathrm{Fe} / \mathrm{O}$ tends to fall steeply in association with smaller values of $\theta_{B n}$. At $100 \mathrm{keV} /$ nucleon, the percentage of $\mathrm{Fe} / \mathrm{O}$ in these interplanetary shocks (IPS) events varies by about a factor of 5 . But, at $2 \mathrm{MeV} /$ nucleon, the variation spans 2 orders of magnitude. Thus, IPS events exhibit the same extreme morphologies as the SEP events [Tylka et al., 2005, Figure 1]. These authors concluded that this fact alone suggests that the variability originates in the details of shock acceleration, and not in two distinct acceleration mechanisms. Present results evidence that the shock front nonstationarity which affects the particle acceleration at shocks front can also be a source of experimental data modulations in addition to those due to the charge state and the shock-normal angle.

[58] We have also reported in Figure 16 the results issued from the test particle simulations obtained by Tylka and Lee [2006] and Sandroos and Vainio [2007] where nonstationary effects of the shock front are excluded (Figures 16c and $16 \mathrm{~d})$. Figures $17 \mathrm{a}$ and $17 \mathrm{~b}$ shows the results issued from our test particle simulations where nonstationary effects are included via the shock front self-reformation. The initial conditions and seed populations used in our simulations are defined as follows. We introduce average abundance ratios $\langle\mathrm{Fe} / \mathrm{O}\rangle \approx 1.08$ for flare component and $\langle\mathrm{Fe} / \mathrm{O}\rangle_{\text {flare }} /\langle\mathrm{Fe} / \mathrm{O}\rangle_{\text {coronal }} \approx 8$ for coronal component, in order to approach the ratio value $\langle\mathrm{Fe} / \mathrm{O}\rangle \approx 1.08$ measured experimentally by satellites [Reames et al., 1994; Reames, 1995]; \langle\rangle is an average over all selected events. The ratio $R=\left\langle\mathrm{O}_{\text {flare }} / \mathrm{O}_{\text {coronal }}\right\rangle$ used by Tylka and Lee [2006] and Sandroos and Vainio [2007] cannot be directly measured, so it is a free parameter which can be varied. The chargestates of flare and coronal components used in our simulations are $Q_{\mathrm{O} \text {,flare }}=8^{+}, Q_{\mathrm{Fe} \text {,flare }}=20^{+}, Q_{\mathrm{O} \text {, coronal }}=6^{+}$, and $Q_{\mathrm{Fe}, \text { coronal }}=10^{+}$, corresponding approximately to the values inferred by Tylka and Lee [2006] and used by Sandroos and Vainio [2007]. The temperature ratio of flare and coronal seeds is 4 , which is close to that $(=3.1)$ used by Tylka and Lee [2006]. Herein, the $\mathrm{Fe} / \mathrm{O}$ ratio spectrum is calculated from the location of the shock overshoot to far upstream (about 260 $\Delta$ away from the overshoot).

[59] Figure 17a shows the energy spectrum of $\mathrm{Fe} / \mathrm{O}$ ratio normalized to the coronal value $=0.134$ which corresponds to Figure 5 of Tylka and Lee [2006] as calculated for different $R$ values with a continuously-time self-reforming shock shown in Figure 1. The key features are the following:

[60] 1. The spectra variations of $\mathrm{Fe} / \mathrm{O}$ ratio obtained for different values of $R=\left\langle\mathrm{O}_{\text {flare }} / \mathrm{O}_{\text {coronal }}\right\rangle$ are similar to the previous results obtained by Tylka and Lee [2006];

[61] 2. In particular, these variations are the strongest for low $R$ values and almost disappear for high $R$ values $(R=50)$ where the $\mathrm{Fe} / \mathrm{O}$ ratio becomes independent of the energy;

[62] 3. For a fixed $R$ value, solid, dashed, and dash-dotted curves represent the results obtained as the $\mathrm{Fe} / \mathrm{O}$ ratio spectrum is time-averaged from $\tilde{t}=628$ to different ending times $\tilde{t}=1552,1600$, and 1744 respectively (as in Section 4). It clearly appears that the variations of the spectrum become even more amplified by the nonstationarity; these amplifications are the strongest for low $R$ values but are smoothed 

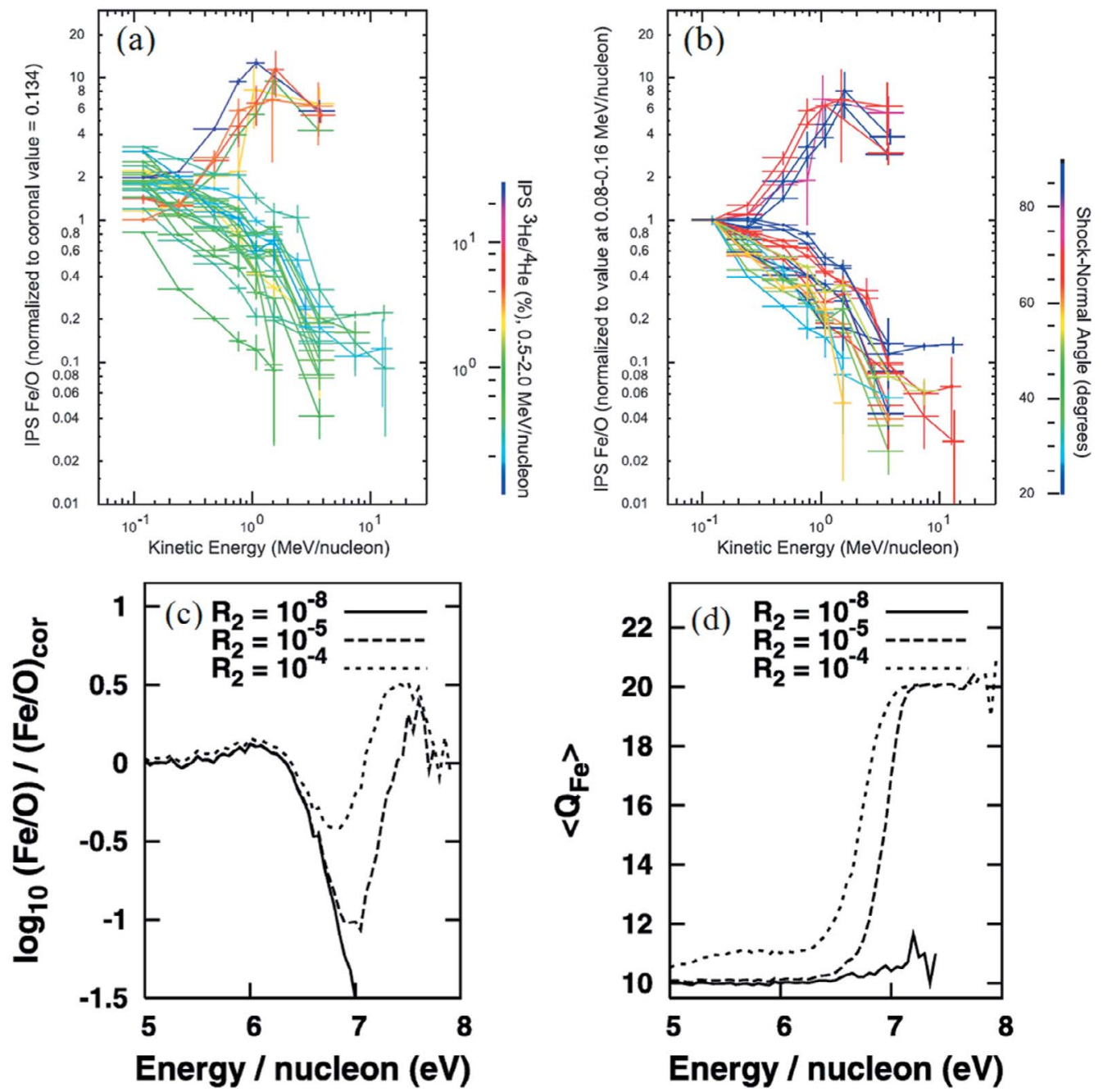

Figure 16. ( $\mathrm{a}$ and $\mathrm{b}$ ) The observational results of $\mathrm{Fe} / \mathrm{O}$ ratio versus energy for 23 traveling interplanetary shock (IPS) events [Desai et al., 2003] in which the energy dependence is strong [from Tylka et al., 2005, Figure 3]. Data points are from ACE ULEIS $\left(\sim 0.1-2.0 \mathrm{MeV}\right.$ nucleon $\left.{ }^{-1}\right)$, Wind LEMT $(\sim 2.5-10 \mathrm{MeV}$ nucleon $\left.^{-1}\right)$, and ACE SIS $\left(10-15 \mathrm{MeV}\right.$ nucleon $\left.^{-1}\right)$. In Figure $16 \mathrm{a}, \mathrm{Fe} / \mathrm{O}$ is normalized to the nominal coronal value (0.134 [Reames, 1995]); the color indicates the ${ }^{3} \mathrm{He} /{ }^{4} \mathrm{He}$ percentage measured in the events within the range $0.5-2.0 \mathrm{MeV}$ nucleon ${ }^{-1}$ from ACE ULEIS [Desai et al., 2003]. Figure 16b shows the percentage of $\mathrm{Fe} / \mathrm{O}$ normalized to the value of each event observed within the range $0.08-0.16 \mathrm{MeV}$ nucleon $^{-1}$; the color indicates the $\theta_{B n}$ value (or its supplement, if smaller) measured from ACE. Figures $16 \mathrm{c}$ and $16 \mathrm{~d}$ show the variation of $\mathrm{Fe} / \mathrm{O}$ ratio and the average charge state of $\mathrm{Fe}$ versus energy, respectively [from Sandroos and Vainio, 2007, Figure 5].

out for high $R$ values. In other words, the shock front nonstationarity has a strong impact on the $\mathrm{Fe} / \mathrm{O}$ ratio spectra.

[63] 4. Figure $17 \mathrm{~b}$ shows the corresponding total average charge states $\left\langle Q_{\mathrm{Fe} \text {,flare }}+Q_{\mathrm{Fe} \text {,coronal }}\right\rangle$ versus energy, which are in good agreement with results of Sandroos and Vainio [2007] reported in Figure 16d. In particular, the variations versus energy only appear for weak $R$ value, but are totally smoothed out for high $R$ value. Moreover, present results show that the nonstationarity of the shock front has a negligible effect on the average charge state of Fe elements whatever the $R$ ratio is; the impact of the $R$ parameter is dominant.

[64] 5. At present, it is difficult to compare more quantitatively our results with previous observational heavy ion spectra [Tylka et al., 2006] because of the lack of infor- mation on initial conditions. In order to fill-in partially this gap, we have performed a parametric simulation analysis versus initial energy. Low initial energy results $\left(\tilde{V}_{t h, \text { flare }} /\right.$ $\left.\tilde{V}_{t h \text {,coronal }}=2 \tilde{V}_{t h p} / V_{t h p}\right)$ are reported in Figures $17 \mathrm{c}$ and $17 \mathrm{~d}$, in order to be compared with the high energy results $\left(\tilde{V}_{t h \text {,flare }} / \tilde{V}_{t h, \text { coronal }}=10 V_{t h p} / 5 \tilde{V}_{t h p}\right)$ shown in Figures $17 \mathrm{a}$ and $17 \mathrm{~b}$. The striking feature is that, for $R=0$, the ratio $\mathrm{Fe} / \mathrm{O}$ is almost independent of the energy range (Figure 17c). Moreover, for finite $R$ values, nonstationary effects lead to some fluctuations forcing the ratio $\mathrm{Fe} / \mathrm{O}$ to follow a larger hump in low initial energy case (Figure 17c), and in contrast a deeper depletion in the high initial energy case (Figure 17a). Moreover, for both initial low and high energy cases, nonstationary effects again amplify the fluctuations in the 

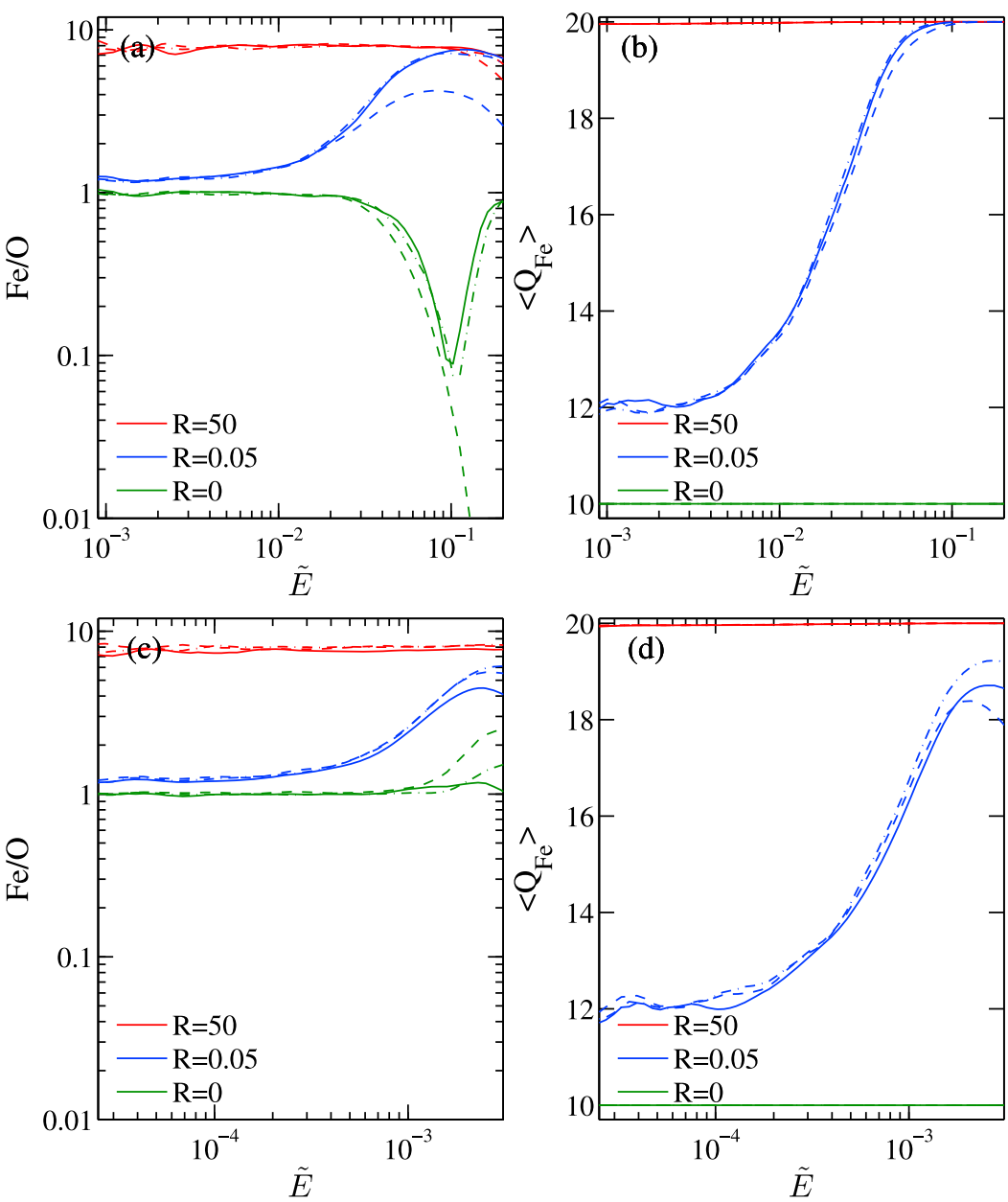

Figure 17. Figures $17 \mathrm{a}$ and $17 \mathrm{~b}$, defined for the high energy case $\tilde{V}_{t h, \text { flare }} / \tilde{V}_{t h \text {,coronal }}=10 \tilde{V}_{t h p} / 5 \tilde{V}_{t h p}$, show our simulation results obtained for a continuously time self-reforming shock (Figure 1). (a) The percentage of the $\mathrm{Fe} / \mathrm{O}$ ratio (normalized to the coronal value $=0.134$ ) versus normalized kinetic energy, for different values of the parameter $R=\left\langle\mathrm{O}_{\text {flare }} / \mathrm{O}_{\text {coronal }}\right\rangle$. For each concerned $R$ value, the solid, dashed, and dash-dotted curves represent the results obtained at $\tilde{t}=1552,1600$, and 1744, respectively. (b) The corresponding average charge state of Fe versus normalized kinetic energy, as calculated for each case of Figure $17 \mathrm{a}$ (left). Figures $17 \mathrm{c}$ and $17 \mathrm{~d}$ are similar to Figures $17 \mathrm{a}$ and $17 \mathrm{~b}$, respectively, and are defined for low energy case $\left(\tilde{V}_{t h, \text { flare }} / \tilde{V}_{t h \text {,coronal }}=2 \tilde{V}_{t h p} / \tilde{V}_{t h p}\right)$.

$\mathrm{Fe} / \mathrm{O}$ energy spectra but have a weaker impact on $\left\langle Q_{\mathrm{Fe}, \text { flare }}{ }^{+}\right.$ $\left.Q_{\mathrm{Fe}, \text { coronal }}\right\rangle$ spectra except for high energy range $(\tilde{E}>0.2$ in Figure 17d).

[65] In summary, nonstationary effects have an important impact on the variation of $\mathrm{Fe} / \mathrm{O}$ ratio versus energy which, sometimes, is comparable to that due to the variation of $R$ ratio; these effects add to those due the particle charge state and shock-normal angle. In contrast, the impact of the nonstationarity is rather limited on the total average charge states whatever the $R$ ratio is.

\section{Conclusions}

[66] In this paper, we have used electric and magnetic field shock profiles produced in a 1-D PIC simulation and test-particle simulations in order to analyze the dynamics and energization of upstream Maxwellian heavy ions interacting with a nonstationary (self-reforming) perpendicular shock. This approach is self-consistent on proton scale but not on heavy ions scales (not included self-consistently), but allows to present the advantage of relative simplicity and to perform good statistics of various species of heavy ions at reasonable computer cost; this point is particularly important for very heavy ions. A parametric analysis has been performed by varying initial thermal energy of the Maxwellian distribution and the charge-mass ratio of different heavy ion species. This analysis has been focused on so called "stationary cases" (equivalent to the fixed shock profiles selected within a self-reformation cycle) and "nonstationary cases" (equivalent to the continuously time-evolving shock profiles covering one or several full self-reformation cycles) and has evidenced the following features:

[67] 1. As for light ions (protons), the incident heavy ions can be separated into two parts: reflected ions (R) and directly transmitted (DT) ions for both stationary and nonstationary cases. Reflected heavy ions suffer different shock acceleration mechanisms SSA and SDA before penetrating the shock front and propagating downstream. The highest 
energy part of the spectrum calculated within the shock front is mainly formed by SDA reflected ions; SSA reflected ions poorly contribute to this high energy part.

[68] 2. The fraction of reflected heavy ions rapidly increases with their initial thermal energies in both stationary and nonstationary cases. However, as the width of the whole shock front is large (and its amplitude decreases) as for profile $\mathrm{B}$, this fraction stays almost constant but its high energy range increases (Figure 3 ). In contrast, the fraction of reflected heavy ions decreases rapidly with the $\mathrm{Q} / \mathrm{M}$ ratio (i.e. for heavier ions), since heavier ions penetrate further downstream without being reflected.

[69] 3. One important feature is the evidence of an injected monoenergetic heavy ion population within the high energy range which is supported by SDA reflected ions. This distribution not only persists quite well in presence of nonstationary effects (for high initial thermal energies), but in addition its amplitude is strongly reinforced by these effects. However, as the ratio $\mathrm{Q} / \mathrm{M}$ increases, the evidence of this distribution is not so clear according to the concerned shock profile. In addition, as the $\mathrm{Q} / \mathrm{M}$ ratio decreases, the nonstationary effects tend to decrease this population (which still persists for all $\mathrm{Q} / \mathrm{M}$ ratios of concern).

[70] 4. Large amplitude front (associated with a steep ramp) as that generated during the self-reformation facilitates the reflection of heavy ions, since the increase of electromagnetic fields at the shock front take place more quickly as compared to the time during which heavy ions interact with steep shock profiles.

[71] 5. The location of heavy ions vortex center around the shock transition region shifts downstream as the chargemass ratio decreases due to the increasing inertial effects which allows heavier ions to penetrate further downstream before (or even without) being reflected. One important point is that this location is independent on the initial thermal energy of heavy ions.

[72] 6. The downstream energy spectra can strongly vary from a Maxwellian to a power law type distribution for different fixed shock profiles. However, the continuously self-reforming shock lead to a power law distribution whose index only slightly varies for different initial $\tilde{V}_{t h i}$.

[73] 7. The evidence of power law in the downstream spectra without invoking diffusive shock acceleration (DSA) is not surprising. The SDA and SSA processes have been already proposed to generate power law energy spectra in regions ranging from downstream to upstream of quasiperpendicular shocks [Zank et al., 1996; Lipatov and Zank, 1999]. Present results show that this feature persists quite well for different heavy ion species and more precisely in the downstream region i.e. where the DSA cannot be invoked. Ideally, the power laws obtained in DSA depend on the shock compression ratio due to the multiple encounter coupling of the particles to the shock compression. Present power laws are presumably due to the thermal spreads of ions and the stochasticity of the fluctuating fields.

[74] 8. Most DT ions contribute to the low and middle energy part of the downstream spectrum, while "old" reflected ions mainly contribute to its high energy part; "old" refer the ions which have been reflected by the shock front before penetrating the downstream region. Moreover, "freshly" reflected ions contribute to the high energy part of the shock front spectrum; "freshly" refer the ions which have just been reflected but are still present in the shock front region. Moreover, our results show that the acceleration "efficiency" of the R heavy ions can be much higher than that deduced from the model of Toida and Ohsawa [1997] (in particular at steep shock profiles), where the contributing energy gain along $x$ direction has been neglected.

[75] 9. The nonstationarity of the shock front has a strong impact on $\mathrm{H}^{+}$and ${ }^{4} \mathrm{He}^{2+}$ ion energy spectra which is comparable to that obtained by varying the percentage of helium ions as investigated by Chapman et al. [2005]. This result only applies for low helium percentage cases ${ }^{4} \mathrm{He}^{2+} \%$ (at least lower than $10 \%$ ) for which the shock front self-reformation stays unchanged and is only controlled by the reflected upstream protons.

[76] 10. Furthermore, the variations of $\mathrm{Fe} / \mathrm{O}$ ratio spectra in high energy part have been retrieved for different $R$ ratio values as initially evidenced experimentally by Tylka et al. [2005], and in test particle simulations of a stationary shock [Tylka and Lee, 2006; Sandroos and Vainio, 2007]. This variation is mainly observed for low $R$ values but disappears for high $R$ values. In addition, present results show that, for low $R$ values, the nonstationary effects of the shock front strongly amplify these variations. This suggests that such effects should be considered in addition to the shock-normal angle and combined seed populations already included in current SEP model and in the interpretation of experimental data. In contrast, these nonstationary effects have a poor impact on the total average charge states.

[77] The present conclusions are based on one-dimensional test particle simulations applied to self-consistent nonstationary perpendicular shock profiles (based on protons dynamics). Further investigation of heavy ions dynamics by using fully self-consistent PIC simulations for both protons and heavy ions is under active investigation, and will be presented in a further work. In addition, we also note that the oblique [Savoini and Lembège, 1994], curved [Savoini and Lembège, 2001], rippled shock front [Lembège and Savoini, 1992; Lembège et al., 2009] and the turbulence upstream and downstream of the shock [Zank et al., 2004; Lu et al., 2009; Giacalone and Decker, 2010; Guo et al., 2010] in high dimensional simulations may also affect particle acceleration. The impact of downstream ion cyclotron wave on particle heating [Lu and Wang, 2005, 2006] could be retrieved in quasi-perpendicular shock simulations with at least two dimensions. Shock reformation is only one aspect of the larger question of how particles initially interact with a realistic shock. Since the self-reformation is a robust process evidenced in 1-D, 2-D and 3-D simulations [Hellinger et al., 2002; Lembège et al., 2009; Shinohara et al., 2011], we choose to focus our attention in the present analysis on the impact of a self-reforming shock, in the most simple condition i.e. for a strictly perpendicular 1-D shock on the dynamics of heavy ions. Further work will present the particle acceleration at 2-D rippled shock front with electromagnetic fluctuations.

[78] Acknowledgments. This research work has been performed while one of the authors (Z.Y.) was staying in France at LATMOS laboratory in Guyancourt (near Paris) which is thanked for its hospitality and the access to computing facilities. The initial one-dimensional PIC simulations have been performed on the supercomputer of IDRIS center located at Orsay (near Paris). This work was partly supported by the National Science 
Foundation of China grants 40725013, 40974083, 40974103, and 41031063, Ocean Public Welfare Scientific Research Project, State Oceanic Administration People's Republic of China (201005017) and the State Scholarship Fund of China Scholarship Council 2009634051.

[79] Philippa Browning thanks the reviewers for their assistance in evaluating this paper.

\section{References}

Axford, W. I., E. Lee, and G. Skadron (1977), The acceleration of cosmic rays by shock waves, Proc. Int. Conf. Cosmic Rays 15th, 11, 132.

Band, D., et al. (1993), Batse observations of gamma-ray burst spectra. I. spectral diversity, Astrophys. J., 413, 281.

Begelman, M., and J. G. Kirk (1990), Shock-drift particle acceleration in superluminal shocks: a model for hot spots in etragalactic radio sources, Astrophys. J., 353, 66.

Bell, A. R. (1978a), The acceleration of cosmic rays in shock front: I, Mon. Not. R. Astrophys. Soc., 182, 147.

Bell, A. R. (1978b), The acceleration of cosmic rays in shock front: II, Mon. Not. R. Astrophys. Soc., 182, 443.

Berezhko, E. G., and H. J. Völk (2007), Spectrum of cosmic rays produced in supernova remnants, Astrophys. J., 661, L175.

Biskamp, D., and H. Welter (1972), Ion heating in high-Mach-number, oblique, collisionless shock waves, Phys. Rev. Lett., 28, 410.

Blandford, R. D., and D. Eichler (1987), Particle acceleration at astrophysical shocks: A theory of cosmic ray origin, Phys. Rep., 154, 1 .

Blandford, R. D., and J. P. Ostriker (1978), Particle acceleration by astrophysical shocks, Astrophys. J., 221, L29.

Chalov, S. V. (2001), Shock drift acceleration of pickup protons at corotating interaction regions, J. Geophys. Res., 106, 18,667.

Chapman, S. C., R. E. Lee, and R. O. Dendy (2005), Perpendicular shock reformation and ion acceleration, Space Sci. Rev., 121, 5.

Cohen, C. M. S., E. C. Stone, R. A. Mewaldt, R. A. Leske, A. C. Cummings, G. M. Mason, M. I. Desai, T. T. von Rosenvinge, and M. E. Wiedenbeck (2005), Heavy ion abundances and spectra from the large solar energetic particle events of October-November 2003, J. Geophys. Res., 110, A09S16, doi:10.1029/2005JA011004.

Decker, R. B. (1983), Formation of shock-spike events at quasi-perpendicular shocks, J. Geophys. Res., 88, 9959.

Decker, R. B. (1988), Computer modeling of test particle acceleration at oblique shocks, Space Sci. Rev., 48, 195.

Decker, R. B., and L. Vlahos (1985), Shock drift acceleration in the presence of waves, J. Geophys. Res., 90, 47.

Decker, R. B., and L. Vlahos (1986), Numerical studies of particle acceleration at turbulent, oblique shocks with an application to prompt ion acceleration during solar flares, Astrophys. J., 306, 710.

Desai, M. I., G. M. Mason, J. R. Dwyer, J. E. Mazur, R. E. Gold, S. M. Krimigis, C. W. Smith, and R. M. Skoug (2003), Evidence for a suprathermal seed population of heavy ions accelerated by interplanetary shocks near $1 \mathrm{AU}$, Astrophys. J., 588, 1149.

Ellison, D. C., and R. Ramaty (1985), Shock acceleration of electrons and ions in solar flares, Astrophys. J., 298, 400.

Giacalone, J. (2004), Large-scale hybrid simulations of particle acceleration at a parallel shock, Astrophys. J., 609, 452.

Giacalone, J., and R. Decker (2010), The original low-energy anomalous cosmic rays at the solar-wind termination shock, Astrophys. J., 710, 91.

Giacalone, J., and J. R. Jokipii (1996), Perpendicular transport in shock acceleration, J. Geophys. Res., 101, 11,095.

Gordon, B. E., M. A. Lee, E. Möbius, and K. J. Trattner (1999), Coupled hydromagnetic wave ecitation and ion acceleration at interplanetary traveling shocks and Earth's bow shock revisited, J. Geophys. Res., $104,28,263$

Guo, F., J. R. Jokipii, and J. Kota (2010), Particle acceleration by collisionless containing large-scale magnetic-field variations, Astrophys. J., $725,128$.

Hada, T., M. Oonishi, B. Lembège, and P. Savoini (2003), Shock front nonstationarity of supercritical perpendicular shocks, J. Geophys. Res., 108(A6), 1233, doi:10.1029/2002JA009339.

Hellinger, P., P. Travnicek, and H. Matsumoto, (2002), Reformation of perpendicular shocks: Hybrid simulations, Geophys. Res. Lett., 29(24), 2234, doi:10.1029/2002GL015915.

Hsieh, K. C., and J. A. Simpson (1970), Galactic ${ }^{3} \mathrm{He}$ above $10 \mathrm{MeV}$ per nucleon and the solar contributions of hydrogen and helium, Astrophys. J., 162, L197.

Hudson, P. D. (1965), Reflection of charged particles by plasma shocks, Mon. Not. R. Astron. Soc., 131, 23.

Kahler, S. (1994), Injection profiles of solar energetic particles as functions of coronal mass ejection heights, Astrophys. J., 428, 837.

Katsouleas, T., and J. M. Dawson (1983), Unlimited electron acceleration in laser-driven plasma waves, Phys. Rev. Lett., 51, 392.
Krymsky, G. F. (1977), A regular mechanism for accelerating charged particles at the shock front, Dokl. Akad. Nauk SSSR, 234, 1306.

Lee, M. A. (1983), Coupled hydromagnetic wave excitation and ion acceleration at interplanetary traveling shocks, J. Geophys. Res., 88, 6109.

Lee, M. A. (1999), The injection, acceleration, and dynamical influence of interstellar pickup ions at the solar wind termination shock, Astrophys. Space Sci., 264, 497.

Lee, M. A., and J. M. Ryan (1986), Time-dependent coronal shock acceleration of energetic solar flare particles, Astrophys. J., 303, 829.

Lee, M. A., V. D. Shapiro, and R. Z. Sagdeev (1996), Pickup ion energization by shock surfing, J. Geophys. Res., 101, 4777.

Lee, R. E., S. C. Chapman, and R. O. Dendy (2004), Numerical simulations of local shock reformation and ion acceleration in supernova remnants, Astrophys. J., 604, 187.

Lee, R. E., S. C. Chapman, and R. O. Dendy (2005), Ion acceleration processes at reforming collisionless shocks, Phys. Plasmas, 12, 012901.

Lembège, B., and J. M. Dawson (1987), Self-consistent study of a perpendicular collisionless and nonresistive shock, Phys. Fluids., 30, 1767.

Lembège, B., and P. Savoini (1992), Nonstationarity of a two-dimensional quasiperpendicular supercritical collisionless shock by self-reformation, Phys. Fluids B, 4, 3533 .

Lembège, B., P. Savoini, P. Hellinger, and P. Travnicek (2009), Nonstationarity of a two-dimensional perpendicular shock: Competing mechanisms, J. Geophys. Res., 114, A03217, doi:10.1029/2008JA013618.

Lever, E. L., K. B. Quest, and V. D. Shapiro (2001), Shock surfing vs. shock drift acceleration, Geophys. Res. Lett., 28, 1367.

Li, G., G. P. Zank, and W. K. M. Rice (2003), Energetic particle acceleration and transport at coronal mass ejection drive shocks, J. Geophys. Res., 108(A2), 1082, doi: 10.1029/2002JA009666.

Lipatov, A. S., and G. P. Zank (1999), Pickup ion acceleration at low- $\beta_{p}$ perpendicular shocks, Phys. Rev. Lett., 82, 3609.

Lipatov, A. S., G. P. Zank, and H. L. Pauls (1998), The acceleration of pickup ions at shock waves: Test particle-mesh simulations, J. Geophys. Res., 103, 29,679.

Lobzin, V. V, V, V, Krasnoselskikh, J-M Bosqued, J-L. Pincon, S. J. Schwartz, and M. Dunlop (2007), Nonstationarity and reformation of high-Mach-number quasiperpendicular shocks: Cluster observations, Geophys. Res. Lett., 34, L05107, doi:10.1029/2006GL029095.

Lu, Q. M., and S. Wang (2005), Formation of $\mathrm{He}^{2+}$ shell-like distributions downstream of the Earth's bow shock, Geophys. Res. Lett., 32, L03111, doi:10.1029/2004GL021508.

Lu, Q. M., and S. Wang (2006), Electromagnetic waves downstream of quasi-perpendicular shocks, J. Geophys. Res., 111, A05204, doi:10.1029/2005JA011319.

Lu, Q. M., Q. Hu, and G. P. Zank (2009), The interaction of Alfvén waves with perpendicular shocks, Astrophys. J., 706, 687.

Malkov, M. A., and L. O'C Drury (2001), Nonlinear theory of diffusive acceleration of particles by shock waves, Rep. Prog. Phys., 64, 429.

Mazelle, C., B. Lembège, A. Morgenthaler, K. Meziane, T. S. Horbury, V. Genot, E. A. Lucek, and I. Dandouras (2010), Self-reformation of the quasi-perpendicular shock: Cluster observations, AIP Conf. Proc., 1216, 471, doi:10.1063/1.3395905.

Morse, D. L., W. W. Destler, and P. L. Auer (1972), Nonstationary behavior of collisionless shocks, Phys. Rev. Lett., 28, 13.

Nishimura, K., H. Matsumoto, H. Kojima, and S. P. Gary (2003), Particle simulation of re-formation at collisionless perpendicular shocks: Coherent behavior of reflected ions, J. Geophys. Res., 108(A5), 1182, doi: 10.1029/ 2002JA009671.

Ng, C. K., D. V. Reames, and A. J. Tylka (2003), Modeling shock-accelerated solar energetic particles coupled to interplanetary Alfvén waves, Astrophys. $J ., 591,461$

Omidi, N., D. Winske, and C. S. Wu (1986), The effect of heavy ions on the formation and structure of cometary bow shocks, Icarus, 66, 165.

Reames, D. V. (1995), Coronal abundances determined from energetic particles, Adv. Space Res., 15, 41.

Reames, D. V. (1999), Particle acceleration at the sun and in the heliosphere, Space Sci. Rev., 90, 413.

Reames, D. V. (2002), Magnetic topology of impulsive and gradual solar energetic particle events, Astrophys. J., 571, L63.

Reames, D. V., J. P. Meyer, and T. T. von Rosenvinge (1994), Energeticparticle abundances in impulsive solar flare events, Astrophys. J. Suppl. Ser., 90, 649 .

Reames, D. V., S. W. Kahler, and C. K. Ng (1997), Spatial and temporal invariance in the spectra of energetic particles in gradual solar events, Astrophys. J., 491, 414

Rice, W. K. M., G. P. Zank, and G. Li (2003), Paricle acceleration and coronal mass ejection driven shocks: Shocks of arbitrary strength, J. Geophys. Res., 108(A10), 1369, doi:10.1029/2002JA009756. 
Sagdeev, R. Z. (1966), Cooperative phenomena and shock waves in collisionless plasmas, Rev. Plasma Phys., 4, 23.

Sandroos, A., and R. Vainio (2007), Simulation results for heavy ion spectral variability in large gradual solar energetic particle events, Astrophys. $J ., 662, \mathrm{~L} 127$.

Savoini, P., and B. Lembège (1994), Electron dynamics in two- and onedimensional oblique supercritical collisionless magnetosonic shocks, J. Geophys. Res., 99, 6609.

Savoini, P., and B. Lembège (2001), Two-dimensional simulations of a curved shock: Self-consistent formation of the electron foreshock, J. Geophys. Res., 106, 12,975.

Schmitz, H., S. C. Chapman, and R. O. Dendy (2002), Electron preacceleartion mechanisms in the foot region of high Alfvénic mach number shocks, Astrophys. J., 579, 327

Scholer, M., and S. Matsukiyo (2004), Nonstationarity of quasi-perpendicular shocks: A comparison of full particle simulations with different ion to electron mass ratio, Ann. Geophys., 22, 2345.

Scholer, M., I. Shinohara, and S. Matsukiyo (2003), Quasi-perpendicular shocks: length scale of the cross-shock potential shock reformation, and implication for shock surfing, J. Geophys. Res., 108(A1), 1014 doi: 10.1029/2002JA009515.

Shalchi, A. (2009), Diffusive shock acceleration in supernova remnants: On the validity of the Bohm limit, Astroparticle Phys., 31, 237.

Shapiro, V. D., and D. Üçer (2003), Shock surfing acceleration, Planet Space Sci., 51, 665.

Shimada, N., and M. Hoshino (2000), Strong electron acceleration at high Mach number shock waves: Simulation study of electron dynamics, Astrophys. J., 543, L67.

Shinohara, I., M. Fujimoto, R. Takaki, and T. Inari (2011), A threedimentional Particle-in-cell simulation of quasi-perpendicular shock on Fujitsu FX-1 Cluster, IEEE Trans. Plasma Sci., 39, 1173, doi:10.1109/ TPS.2011.2106515.

Toida, M., and Y. Ohsawa (1995), Heavy ion acceleration by nonlinear magnetosonic waves in a two-ion-species plasma, J. Phys. Soc. Jpn., 64, 2036.

Toida, M., and Y. Ohsawa (1997), Simulation studies of acceleration of heavy ions and their elemental compositions, Sol. Phys. Jpn., 171, 161

Tylka, A. J., and M. A. Lee (2006), A model for spectral and compositional variability at high energies in large, gradual solar particle events, Astrophys. J., 646, 1319.

Tylka, A. J., C. M. S. Cohen, W. F. Dietrich, M. A. Lee, C. G. Maclennan, R. A. Mewaldt, C. K. Ng, and D. V. Reames (2005), Shock geometry, seed populations, and the origin of variable elemental composition at high energies in large gradual solar particle events, Astrophys. J., 625, 474.
Tylka, A. J., C. M. S. Cohen, W. F. Dietrich, M. A. Lee, C. G. Maclennan, R. A. Mewaldt, C. K. Ng, and D. V. Reames (2006), A comparative study of ion characteristics in the large gradual solar energetic particle events of 2002 April 21 and 2002 August 24, Astrophys. J. Suppl. Ser., 164, 536.

Üçer, D., and V. D. Shapiro (2001), Unlimited relativistic shock surfing acceleration, Phys. Rev. Lett., 87, 075001.

Üçer, D., and V. D. Shapiro (2005), Relativistic surfing acceleration of ions at oblique shocks, Phys. Lett. A, 346, 163.

Wang, Y. M., M. Pick, and G. M. Mason (2006), Coronal holes, jets, and the origin of 3he-rich particle events, Astrophys. J., 639, 495.

Webb, G. M., W. I. Axford, and T. Terasawa (1983), On the drift mechanism for energetic charged particles at shocks, Astrophys. J., 270, 537.

Webb, G. M., G. P. Zank, M. Ko, and D. J. Donohue (1995), Multidimentional green's functions and the statistics of diffusive shock acceleration, Astrophys. J., 453, 178

Yang, Z. W., Q. M. Lu, B. Lembège, and S. Wang (2009a), Shock front nonstationarity and ion acceleration in supercritical perpendicular shocks, J. Geophys. Res., 114, A03111, doi: 10.1029/2008JA013785.

Yang, Z. W., Q. M. Lu, and S. Wang (2009b), The evolution of the electric field at a nonstationary perpendicular shock, Phys. Plasmas, 16, 1.

Yuan, X. Q., I. H. Cairns, L. Trichtchenko, R. Rankin, and D. W. Danskin (2009), Confirmation of quasi-perpendicular shock reformation in twodimensional hybrid simulations, Geophys. Res. Lett., 36, L05103, doi: 10.1029/2008GL036675.

Zank, G. P., H. L. Pauls, I. H. Cairns, and G. M. Webb (1996), Interstellar pickup ions and quasi-perpendicular shocks: Implications for the termination shock and interplanetary shocks, J. Geophys. Res., 101, 457.

Zank, G. P., W. K. M. Rice, and C. C. Wu (2000), Particle acceleration and coronal mass ejection driven shocks: A theoretical model, J. Geophys. Res., 105, 25,079.

Zank, G. P., G. Li, V. Florinski, W. H. Matthaeus, G. M. Webb, and J. A. le Roux (2004), Perpendicular diffusion coefficient for charged particles of arbitrary energy, J. Geophys. Res., 109, A04107, doi:10.1029/ 2003JA010301.

Zank, G. P., G. Li, V. Florinski, Q. Hu, D. Lario, and C. W. Smith (2006), Particle acceleration at perpendicular shock waves: Model and observations, J. Geophys. Res., 111, A06108, doi:10.1029/2005JA011524.

B. Lembège, LATMOS-UVSQ-IPSL-CNRS, Guyancourt F-78280, France.

Q. M. Lu and Z. W. Yang, CAS Key Laboratory of Basic Plasma Physics, School of Earth and Space Sciences, University of Science and Technology of China, Hefei, Anhui 230026, China. (qmlu@ustc.edu.cn) 